\title{
Iterated Revision and Minimal Change of Conditional Beliefs
}

\author{
Craig Boutilier \\ University of British Columbia
}

\begin{abstract}
We describe a model of iterated belief revision that extends the AGM theory of revision to account for the effect of a revision on the conditional beliefs of an agent. In particular, this model ensures that an agent makes as few changes as possible to the conditional component of its belief set. Adopting the Ramsey test, minimal conditional revision provides acceptance conditions for arbitrary right-nested conditionals. We show that problem of determining acceptance of any such nested conditional can be reduced to acceptance tests for unnested conditionals. Thus, iterated revision can be accomplished in a "virtual" manner, using uniterated revision.
\end{abstract}

\section{Introduction}

The acceptance conditions for subjunctive conditionals have been widely studied, but no criterion has drawn more attention recently than the Ramsey test. Supposing an agent to possess some belief set $K$, it is instructed by Stalnaker to accept the conditional $A>B$ iff it satisfies the following test:

First add the antecedent (hypothetically) to your stock of beliefs; second make whatever adjustments are required to maintain consistency (without modifying the hypothetical belief in the antecedent); finally, consider whether or not the consequent is true. [32, p.44]

The crucial step in the Ramsey test is the revision of the belief set. The notion of revision adopted will determine which conditionals are accepted and rejected. Conversely, given a fixed (complete) set of accepted conditionals, the revision function adopted by an agent will also be determined.

The AGM theory of revision due to Alchourrón, Gärdenfors and Makinson [2] provides guidelines for the revision of objective belief sets (containing no conditional sentences). These are usually presented as postulates that constrain the behavior of revision functions. We use $K_{A}^{*}$ to denote the 
belief set resulting from the revision of $K$ by $A$. Unfortunately, the theory does not address the issue of revising conditional belief sets. Gärdenfors [14] proposes that belief sets be extended to include conditional beliefs, requiring only that the following postulate, reflecting the Ramsey test, be respected: ${ }^{1}$

(RT) $A>B \in K$ iff $B \in K_{A}^{*}$.

This description of the revision of conditional belief sets says nothing about the preservation of conditionals in a belief set during the course of revision. A hallmark of the AGM theory is its commitment to the principle of informational economy: beliefs are only given up when there are no less entrenched candidates. Yet this principle is ignored in this formulation of conditional belief revision, as well as many related proposals. Given an ordering of entrenchment and belief set $K$, the AGM theory will determine the form of $K_{A}^{*}$. The extended theory, in contrast, when applied to a conditional belief set, imposes almost no constraints on the conditionals deemed acceptable in the new belief set. This has a tremendous impact on the analysis of revision sequences. Should an agent revise its belief set $K$ by $A$, its conditionals $A>B$ determine the form of $K_{A}^{*}$. However, if a revision function imposes no constraints on subsequently accepted conditionals, there can be no logical constraints on subsequent revision of $K_{A}^{*}{ }^{2}$ The fact that $K_{A}^{*}$ was reached by revision of $K$ becomes meaningless.

In this paper, we extend the AGM model of revision to account for such sequences of belief change. We take the epistemic state of an agent to consist of both objective beliefs and (unnested) conditional beliefs. These conditional beliefs can be viewed as capturing the relative degrees of entrenchment of objective beliefs, or equivalently, as completely specifying the agent's revision policies. Acceptance of a new belief forces a change in the agent's objective belief set in accordance with the AGM theory. This necessarily requires some change in the agent's revision policies, or conditional beliefs. We describe how this change can be effected semantically by minimally changing the agent's judgements of the relative entrenchment of objective beliefs. This in turn has the effect of changing as few conditional beliefs as possible consistent with the dictates of the AGM theory (when restricted to the objective component of the belief set). Thus, the original conditional belief set uniquely determines the revised conditional belief set. In particular, from a set of simple, unnested conditionals, the effects

\footnotetext{
${ }^{1}$ Of course, Gärdenfors points out that, given the other constraints of the AGM theory (even weakened somewhat), such a condition is untenable. This celebrated triviality result has been devoted considerable attention. But very compelling arguments (e.g., $[23,27]$ ) have been put forth suggesting that the postulates of the AGM theory should not hold in the case of nonobjective beliefs. This is the point of view adopted here, as we elaborate below.

${ }^{2}$ At least, there can be no constraints other than those imposed by the objective part of $K_{A}^{*}$.
} 
of arbitrary sequences of revisions can be computed. We will demonstrate an especially interesting consequence of this result, namely, that any right-nested conditional arbitrary depth can be effectively reduced to an unnested conditional with the same acceptance conditions.

\section{A Semantic Model for AGM Revision}

We assume an agent to have a deductively closed set of beliefs $K$ taken from some underlying language. For concreteness, we will assume this language to be that of classical propositional logic $\mathrm{L}_{C P L}$, generated by some set of variables $\mathbf{P}$, and with an associated consequence operation $C n$. The identically true and false propositions are denoted $\top$ and $\perp$, respectively. We say $K$ is finitely specifiable if $K=C n(S)$ for some finite set of premises $S$. The expansion of $K$ by new information $A$ is the belief set $K_{A}^{+}=C n(K \cup\{A\})$. The revision of $K$ by $A$ is denoted $K_{A}^{*}$. The process of revision requires some care, for $A$ may contradict elements of $K$. Alchourrón, Gärdenfors and Makinson [2] propose a method for logically delimiting the scope of acceptable revisions. To this end, the AGM postulates below, are maintained to hold for any reasonable notion of revision [15].

(R1) $K_{A}^{*}$ is a belief set (i.e. deductively closed).

(R2) $A \in K_{A}^{*}$.

(R3) $K_{A}^{*} \subseteq K_{A}^{+}$.

(R4) If $\neg A \notin K$ then $K_{A}^{+} \subseteq K_{A}^{*}$.

(R5) $K_{A}^{*}=C n(\perp)$ iff $\mid=-A$.

(R6) If $\mid=A \equiv B$ then $K_{A}^{*}=K_{B}^{*}$.

(R7) $K_{A \wedge B}^{*} \subseteq\left(K_{A}^{*}\right)_{B}^{+}$.

(R8) If $\neg B \notin K_{A}^{*}$ then $\left(K_{A}^{*}\right)_{B}^{+} \subseteq K_{A \wedge B}^{*}$.

An alternative model of revision is based on the notion of epistemic entrenchment $[15,16]$. Given a belief set $K$, we can characterize the revision of $K$ by ordering beliefs according to our willingness to give them up when some contradictory information requires such. If one of two beliefs must be retracted in order to accommodate some new fact, the less entrenched belief will be relinquished, while the more entrenched persists. Gärdenfors [15] presents five postulates for such an ordering and shows that these orderings determine exactly the space of revision functions satisfying the AGM 
postulates. We let $B \leq_{E} A$ denote the fact that $A$ is at least as entrenched as $B$ in theory $K$. A complete set of sentences of this form is sufficient to specify a revision function. We note that the dual of an entrenchment ordering is a plausibility ordering on sentences. A sentence $A$ is more plausible than $B$ just when $\neg A$ is less entrenched than $\neg B$, and means that $A$ would be more readily accepted than $B$ if the opportunity arose. Grove [19] studied this relationship and its connection to the AGM theory.

\subsection{A Semantic Model}

Our extension of the AGM theory will be based on a slightly different semantic representation of revision functions. Grove [19] observed that an entrenchment relation over sentences can be represented using a plausibility ordering over models. Our semantics captures this intuition, though it is (cosmetically) distinguished by the use of an explicit ranking function rather than Grove's system of spheres. In what follows, we describe our basic semantic models and the truth conditions for a number of connectives. While we do not give details here, we note that this system can be axiomatized in using a simple modal logic $\mathrm{CO}^{*}$, and that each of the connectives and semantic restrictions described below can be represented in this logic. We refer to $[8,9,5]$ for details of this type.

We imagine an agent to have some objective belief set $K \subseteq \mathrm{L}_{C P L}$ as well as a collection of revision policies to guide changes of this belief set. A revision model is a triple $M=\langle W, \leq, \varphi\rangle$ intended to capture both aspects of the agent's epistemic state. $W$ is a set of worlds and $\varphi: \mathbf{P} \rightarrow 2^{W}$ is a valuation mapping, assigning atoms to the subsets of worlds at which they hold. The truth of an arbitrary proposition at any world is defined in the usual way. We write $M=_{w} A$ to indicate that $A$ holds at world $w$, and denote by $\|A\|$ the set of such $A$-worlds ( $M$ is usually understood). We also us this notation for sets of sentences $S,\|S\|$ denoting those worlds satisfying each element of $S$, and somewhat loosely refer to worlds falsifying some element of $S$ as $-S$-worlds.

Finally, $\leq$ is a binary relation over $W$. We insist that $\leq$ be transitive and connected. ${ }^{3}$ The interpretation of $\leq$ is as follows: $v \leq w$ iff $v$ is as at least as plausible a state of affairs as $w$. Plausibility is a pragmatic measure that reflects the degree to which one would accept $w$ as a possible state of affairs. If $v$ is more plausible than $w$, loosely speaking $v$ is "more consistent" with the agent's beliefs than $w$. Since $\leq$ is a "total preorder," $W$ is partitioned into $\leq$-equivalence classes, or clusters of equally plausible worlds. These cluster are themselves totally ordered by $\leq$. Thus, $\leq$ can be

\footnotetext{
${ }^{3} \leq$ is (totally) connected if $w \leq v$ or $v \leq w$ for any $v, w \in W$ (this implies reflexivity). This restriction is relaxed in [9], where we develop a weaker logic based on a reflexive, transitive accessibility relation.
} 
viewed as a qualitative ranking relation, assigning to each world a degree of plausibility. Figure 1 illustrates the structure of such an ordering.

We require that epistemically possible worlds be more plausible than epistemically impossible worlds, and that all epistemically possible worlds are equally plausible. In other words, $K$-worlds should be exactly those minimal in $\leq$ :

$$
w \leq v \text { for all } v \in W \text { iff } M \models K
$$

Such models are called $K$-revision models and have as their minimal cluster the set $\|K\|$ (see Figure 1). ${ }^{4}$ Although this is sufficient structure to define a reasonable revision function, we adopt two more restrictions. First, we insist that $K$-revision models are "complete;"that is, every propositional valuation is associated with some element of $W$. In other words, the valuations induced by $\varphi$ and associated with each $w \in W$ must cover the entire range of logical possibilities. This ensures that an agent accords every possibility some degree of plausibility. Second, we assume that each model satisfies the following (restricted) well-foundedness condition.

Definition 1 Let $M=\langle W, \leq, \varphi\rangle$ be a CO-model. For any $A \in \mathrm{L}_{C P L}$ we define

$$
\min (M, A)=\left\{w \in W: M \models_{w} A, \text { and } M=_{v} A \text { implies } w \leq v \text { for all } v \in W\right\}
$$

A model is well-founded iff, for all $A, \min (A, M) \neq \emptyset$.

We are now in a position to define a conditional connective and associated belief revision function (suitable for $K$ ). When a new belief $A$ is accepted, the agent should adopt the set of most plausible $A$-worlds, $\min (M, A)$, as its set of newly epistemically possible situations. The conditional sentence $A>B$ asserts that "If the agent were to adopt belief in $A$, it would believe $B$." The acceptance of such a conditional relative to $M$ is specified as follows: ${ }^{5}$

$$
M \models A>B \text { iff } \min (M, A) \subseteq\|B\|
$$

The Ramsey equivalence immediately provides us with the revision function induced by $M$ (w.r.t.

\footnotetext{
${ }^{4}$ If $K$ is finitely specifiable then this constraint can be expressed in language of CO. Intuitively, this corresponds to the notion of "only knowing" $K$ : all and only $K$-worlds are epistemically possible [22, 5].

${ }^{5}$ The acceptance condition can be specified in a way that does not presume the well-foundedness restriction, and the equivalence to the AGM theory described below is unaffected. Thus, the logic $\mathrm{CO}^{*}$ provides a mechanism for expressing with unary modal connectives the truth conditions proposed by Lewis [24] to avoid the Limit Assumption [9]. However, the representation result of Grove ensures that the class of well-founded models is sufficient to capture all AGM revision functions.
} 


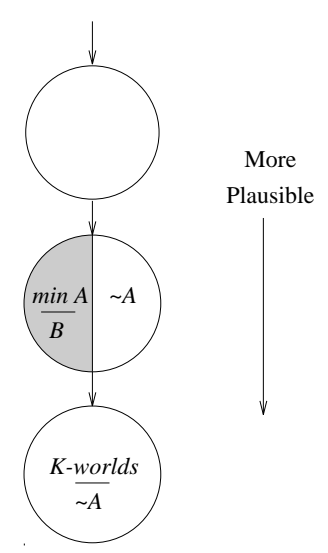

Figure 1: Truth conditions for the conditional $A>B$

$K)$. For any $A \in \mathbf{L}_{C P L}$ we define

$$
K_{A}^{*^{\mathrm{M}}}=\left\{B \in \mathbf{L}_{C P L}: M \models A>B\right\} .
$$

We can show that $*$ satisfies the AGM postulates for belief revision and any AGM revision operator has an equivalent formulation as such a $*$.

Theorem 1 [9] Let $M$ be a $K$-revision model and $*^{\mathrm{M}}$ the revision function determined by $M$. Then $*{ }^{\mathrm{M}}$ satisfies postulates $(R 1)$ through (R8).

Theorem 2 [9] Let * be a revision function satisfying postulates $(R 1)$ through (R8). For any theory $K$ there exists a $K$-revision model $M$ such that $K_{A}^{*}=K_{A}^{*}$ for all $A$.

We call $A>B$ a simple conditional iff $A, B \in \mathbf{L}_{C P L}$. Such unnested conditionals are especially interesting since they alone determine the revision function $*^{\mathrm{M}}$.

In Figure 1, we have a typical $K$-revision model with each large circle representing a cluster of mutually accessible (equally plausible) worlds, with arrows indicating accessibility between clusters. The minimal cluster consists of all $K$-worlds, and we have that $K \vdash \neg A$. The set of minimal $A$-worlds is indicated by the shaded region, and this set forms the set of "newly possible" worlds when $K$ is revised by $A$. Thus $A>B$ should hold just when $B$ is true at each world in the shaded region. 


\subsection{Extended Belief Sets}

We will have occasion to use several other connectives whose semantics we introduce here. First, we define a modality for belief, reading $\mathrm{B} \alpha$ as " $\alpha$ is believed:"

$$
M \models \mathrm{B} \alpha \quad \text { iff } \min (M) \subseteq\|\alpha\| \quad \text { iff } \quad M \models \top>\alpha
$$

This holds just when $\alpha$ is true at each epistemically possible world, those minimal in the plausibility ordering $\leq$. We note that B behaves according to the usual weak S5 interpretation of belief [5].

Statements regarding the relative entrenchment and plausibility of various propositions can also be expressed. We write $B \leq_{E} A$ to denote that $A$ is at least as entrenched as $B$, meaning that $A$ is at least as strongly held a belief as $B$. We have

$$
M \mid=B \leq_{E} A \quad \text { iff } \min (M,-B) \leq \min (M, \neg A)
$$

The dual notion of plausibility, where $B \leq_{P} A$ denotes the fact that $A$ is at least as plausible as $B$, is captured by

$$
M \models B \leq_{P} A \quad \text { iff } \quad \min (M, A) \leq \min (M, B) \quad \text { iff } \quad M \mid=-A \leq_{E} \neg B
$$

For any revision model, we define the objective belief set associated with it to be those propositional sentences that are "believed" in the model, namely.

$$
\left\{\alpha \in \mathbf{L}_{C P L}: M \mid \mathrm{B} \alpha\right\}
$$

We will sometimes refer to this as the propositional belief set or simply the belief set for $M$. Naturally, the belief set for any $K$-revision model is just $K$. We will be more interested here in "subjective beliefs" associated with a revision model, those sentences that are believed, but contain modal operators. Of particular concern are those conditionals that are believed by an agent. We therefore extend the notion of belief set to cover arbitrary sentences. We denote by $\mathbf{L}$ the full language that includes the connectives $>, \mathrm{B}, \leq_{E}$, and any other connectives that we introduce. ${ }^{6}$ The extended belief set associated with $M$ is

$$
\{\alpha \in \mathrm{L}: M \mid \mathrm{B} \alpha\}
$$

\footnotetext{
${ }^{6}$ Technically, we can take this to be the modal language of $\mathrm{CO}^{*}$, in which all such connectives are defined.
} 
We note the following properties of belief sets and extended belief sets. Clearly, for any $K$ revision model we have $K \subseteq E$. Furthermore, extended sets are stable sets of beliefs in the sense of Stalnaker [33]:

Proposition 3 Let $E$ be the extended set determined by $M$. Then: a) $A \in E$ iff $\mathrm{B} A \in E$; $b) A \notin E$ iff $\neg \mathrm{B} A \in E$; and c) $E$ is closed under tautological consequence.

This is a reasonable requirement on the belief set of any rational agent with full powers of introspection. Given this introspective ability, we expect an agent to be able to completely determine the beliefs contained in any revised belief set $K_{A}^{*}$. While the Ramsey test ensures that simple conditionals $A>B$ will be in $E$ if $B \in K_{A}^{*}$, we have an even stronger property: if a conditional fails the Ramsey test its negation is believed.

Proposition 4 Let $E$ be the extended set determined by $M$. Then for any simple conditional $A>B$, either $A>B \in E$ or $\neg(A>B) \in E$.

We refer to this property as the completeness of conditional beliefs.

We note that the connectives B and > have "global" truth conditions. By this we mean that if, say, $A>B$ holds at some world in a model $M$, then it holds at all worlds in $M$. Its acceptance does not depend on the world at which it is being evaluated, but rather on the global (rather than local) structure of $M$. This has important consequences when we consider the meaningfulness of iterated belief and conditional statements in extended sets. For instance, we immediately have that $\mathrm{B}(A>B) \in E$ iff $A>B \in E$. Thus, extended sets provide a mechanism for incorporating both objective beliefs and belief in simple conditionals into a single collection. However, the global nature of acceptance conditions ensures that non-simple conditionals add nothing of interest to the language.

Let us dub any sentence such that all atomic variables lie within the scope of a modality B or a conditional $>$ a subjective sentence. We concentrate on the case of conditionals whose consequent is subjective. It is easy to see that the truth conditions for any conditional $A>\alpha$, for any purely subjective sentence $\alpha$, are uninteresting. As long as $A$ is satisfiable, $A>\alpha$ holds iff $\alpha$ does. Thus, this model of the AGM postulates is suitable only for objective belief sets. For example, we have that $M \mid=A>(B>C)$ iff $\min (M, A) \subseteq\|B>C\|$ iff $M=B>C$. This is certainly not a reasonable property of extended belief sets or revision models. In particular, if the Ramsey test is taken seriously this semantics is not suitable for iterated revision. However, it can be easily extended. 


\section{Minimal Conditional Revision}

\subsection{The Problem of Iterated Revision}

Suppose an agent possesses a belief set $K$. Its revision policies and judgements of entrenchment are captured by the $K$-revision model $M$. If the agent is curious about the status of proposition $B$ after an arbitrary propositional revision $A$, it may simply consider whether $B \in K_{A}^{*}$. In other words, for objective formulae $B$ simply asking whether $B$ is true at each world in $\min (M, A)$ is appropriate, and is sufficient (via the Ramsey test) to determine acceptance of the conditional $A>B$. While this characterization of $K_{A}^{*}$ is clear, it is less obvious just what form the revised extended belief set, $E_{A}^{*}$, should take. As made clear above, the new subjective beliefs of the agent cannot be captured by the set of worlds $\min (M, A)$. If it were, an agent could drastically change its objective belief set $K$ without altering its conditional beliefs, its nested beliefs, or its judgements of entrenchment.

It should be clear why this difficulty arises. The model $M$ is a $K$-revision model, suitable for modeling the revision of belief set $K$, and for determining the truth of conditionals relative to $K$. When the agent's objective beliefs are revised, giving rise to belief set $K_{A}^{*}$, such a model is no longer adequate as a representation of the agent's epistemic state. It cannot retain the same conditionals given a different belief set. Recall, the Ramsey test provides acceptance conditions for conditionals, not truth conditions; and acceptance is relative to a given state of belief. Similarly, if the agent's beliefs change, so too must its "nested" beliefs and its judgements of entrenchment and plausibility. Thus, the $K$-revision model must be given up. The agent must adopt a revised model of its epistemic state, which we denote $M_{A}^{*}$. It is this model that determines the agent's revised extended belief set $E_{A}^{*} \cdot 7$

What are the natural requirements on the new model $M_{A}^{*}$ ? Since $K_{A}^{*}$ is uniquely determined by $M$ we insist that $K_{A}^{*} \subseteq E_{A}^{*}$ and that $K_{A}^{*}$ form the entire objective component of $E_{A}^{*}$. That is, $M_{A}^{*}$ should be a $K_{A}^{*}$-revision model, or that $K_{A}^{*}$ is only known in $M_{A}^{*}$. The minimal cluster of worlds in $M_{A}^{*}$ should be exactly $\min (M, A)$. This is illustrated in Figure 2 . Let us dub this constraint the Basic Requirement on revision functions as applied to models.

The Basic Requirement: If $M$ is a $K$-revision model determining revision function $*$, then the revision model $M_{A}^{*}$ must be such that $\min \left(M_{A}^{*}, \top\right)=\min (M, A)$.

In fact, from a purely logical perspective, this is probably all we want to say about $M_{A}^{*}$. If one changes an objective belief, it is impossible in general to predict what becomes of one's conditionals.

\footnotetext{
${ }^{7}$ Similar observations have been made by Levi [23], Rott [29] and Hansson [21].
} 


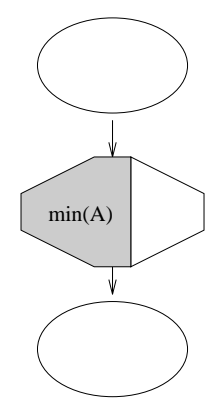

$M$

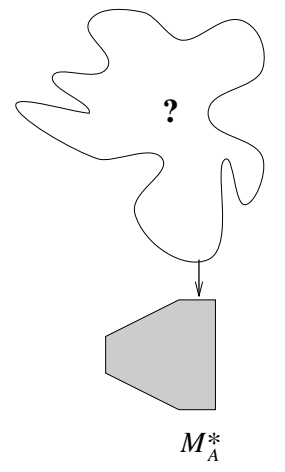

(b)

Figure 2: General constraints on the mapping to a new revision model

This model of iterated revision is captured by Gärdenfors's [15] belief revision systems, although not in this semantic fashion. Such a system consists of a set $\mathbf{K}$ of belief sets and a revision function $*$ that maps $\langle K, A\rangle$, where $K \in \mathbf{K}$ and $A \in \mathbf{L}_{C P L}$, to $K_{A}^{*} \in \mathbf{K}$. The function $*$ must satisfy the AGM postulates, but the behavior of $*$ on the extended belief set $E$ associated (by the Ramsey test) with $K$ is left unspecified. Only postulates (R7) and (R8) constrain how revision of $K_{A}^{*}$ should take place, and these constraints are quite mild. This model provides no guidance as to what conditionals in $E$ should be accepted or rejected in $E_{A}^{*}$. Put another way, no hints are provided on how to revise $K_{A}^{*}$, given its "origination" as a revision of $K$ by $A$. The ordering of worlds in the nebulous region of Figure 2 (b) is completely unspecified.

Belief revision systems of the Gärdenfors type possess two unattractive features, one logical and one pragmatic. First, epistemic states are distinguished solely by their objective component. For any $K$, the extended belief set $E$ associated with $K$ (via the Ramsey test) is fixed, since $K_{A}^{*}$ is fixed. There can be no two distinct extended belief sets that share the same objective part. In other words, there can only be one way of revising a belief set. This is certainly an unnatural restriction, one that is not imposed by our notion of $K$-revision models. There exists a multitude of $K$-revision models for any fixed $K$, reflecting the view that conditionals and judgements of entrenchment form an integral part of an agent's epistemic state. An agent can be in two different belief states, but have the same propositional beliefs in each and differ only on its accepted conditionals. This is a natural extension of revision systems, but it is also quite straightforward. The semantic "characterization" of such a system is completely captured by Figure 2. The general method of Rott [28] is based on the same considerations. He identifies the epistemic state of an agent with an ordering of entrenchment over 
$\mathbf{L}_{C P L}$, capturing exactly the same information as a revision model. Hansson [21] also associates potentially distinct revision functions with equivalent belief sets. ${ }^{8}$

The second criticism is of a more pragmatic nature and is the point we address in the rest of the paper. Everything above points to a mapping of $M$ to $M_{A}^{*}$ satisfying one condition, that the objective belief set associated with $M_{A}^{*}$ be precisely the set $K_{A}^{*}$ determined by $M$. As Figure 2 illustrates, just about all of the ordering information, capturing an agent's conditional beliefs and judgements of entrenchment, is (potentially) lost in this mapping. There is something unsatisfying about this model. The ordering relation $\leq$ is intended to reflect the informational content or importance of beliefs. When certain beliefs must be given up, it seems reasonable to try to keep not only important objective beliefs, but as much of the ordering a possible. Revision should not generally change one's opinion of the relative importance of most sentences.

\subsection{The Semantics of Minimal Conditional Revision}

Instead of arbitrary mappings from $M$ to $M_{A}^{*}$, we will propose a class of natural mappings that preserve as much ordering information as can be expected. This determines the class of minimal conditional revision functions, that tend to preserve the entrenchment information and conditional beliefs found in an extended belief set. It is important to note that the model we propose is not completely general, for it permits only a subset of those revision functions (on extended sets) allowed by the arbitrary mappings described above. However, it is a very natural subset, for it extends the notion of minimal change to subjective formulae. Furthermore, it is suitable for determining the result of propositional revision sequences, or the acceptance of right-nested conditionals, when the general model has little to offer.

The conditionals accepted by an agent are determined by its ordering of plausibility. If we insist that revision preserve as much of this ordering as possible, then, for the most part, the relative entrenchment and plausibility of sentences (hence conditional beliefs) will remain intact. Let $M=\langle W, \leq, \varphi\rangle$ be the revision model reflecting some extended belief set $E$. Given a propositional revision $A$ of $E$ (or the associated $K$ ), we must find a revision model $M_{A}^{*}=\left\langle W, \leq^{\prime}, \varphi\right\rangle$ such that $\leq^{\prime}$ reflects the minimal mutilation of $\leq$.

If $w \in \min (M, A)$, by the Basic Requirement $w$ must be minimal in $\leq^{\prime}$, and these must be the only minimal worlds in $\leq^{\prime}$. For any such $w$ the relationships $w \leq^{\prime} v$ and $v \leq^{\prime} w$ are completely determined by membership of $v$ in $\min (M, A)$, independently of their relationship in $\leq$. Figure 2

\footnotetext{
${ }^{8}$ We discuss the relationship with these systems further in the concluding section.
} 


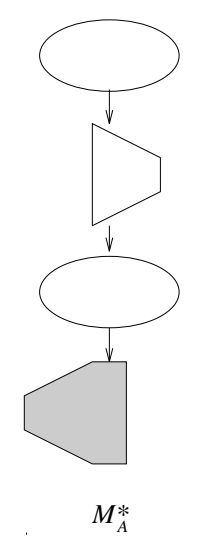

Figure 3: The minimal conditional revision of a model.

illustrates this. For $w, v$ not in $\min (M, A)$, this picture leaves $v \leq w$ completely unspecified. If $\leq$ is to be left intact to the largest possible extent then the most compelling specification is to insist that $w \leq^{\prime} v$ iff $w \leq v$. This has the effect of leaving $\leq$ unaltered except as indisputably required by the Basic Requirement. Such a move, applied to the original model in Figure 2, is illustrated in Figure 3. We dub such a mapping on revision models the minimal conditional revision operator, and describe the revision function it induces on the associated belief and extended belief sets.

Definition 2 Let $M=\langle W, \leq, \varphi\rangle$ be a revision model. The minimal conditional revision operator (or MC-revision operator) * maps $M$ into $M_{A}^{*}$, for any $A \in \mathrm{L}_{C P L}$, where $M_{A}^{*}=\left\langle W, \leq^{\prime}, \varphi\right\rangle$, and: a) if $v \in \min (M, A)$ then $v \leq^{\prime} w$ for all $w \in W$ and $w \leq^{\prime} v$ iff $w \in \min (M, A)$; and b) if $v, w \notin \min (M, A)$ then $w \leq^{\prime} v$ iff $w \leq v$.

Definition 3 Let $E$ be the extended belief set associated with the revision model $M$. The minimal conditional revision function associated with $M$ is $*$, defined for all $A \in \mathbf{L}_{C P L}$ as follows:

$$
E_{A}^{*}=\left\{B \in \mathbf{L}: M_{A}^{*} \mid=\mathrm{B} B\right\}
$$

Let $K \subseteq E$ be the objective component of $E$. The minimal conditional revision (or MCrevision) of $K$, denoted $K_{A}^{*}$, is the restriction of $E_{A}^{*}$ to $\mathrm{L}_{C P L}$; that is

$$
K_{A}^{*}=\left\{B \in \mathrm{L}_{C P L}: M_{A}^{*} \models \mathrm{B} B\right\}
$$


The MC-revision function is simply the AGM operator determined by $M$, when restricted to $K$.

Proposition 5 Let $M$ be a revision model for $K, *{ }^{\mathrm{M}}$ be the AGM revision function determined by $M$ (as given by Definition 1), and $*$ be the $M C$-revision function determined by $M$. For any $A \in \mathbf{L}_{C P L}$, we have $K_{A}^{*}{ }^{\mathrm{M}}=K_{A}^{*}$.

Notice that this enhances the AGM model of revision. The revised extended set $E_{A}^{*}$ is defined using the updated revision model $M_{A}^{*}$ and incorporates subjective beliefs, such as conditionals, statements of entrenchment and plausibility, and nested belief sentences.

If we are to extend the Ramsey test to include nested conditionals, the truth conditions for statements $A>\beta$ must be recast in this framework for subjective $\beta$. For $M$ to satisfy $A>\beta$, we must have $\beta \in E_{A}^{*}$. If $\beta \in \mathbf{L}_{C P L}$, given Proposition 5, these truth conditions will be identical to those provided in Section 2. Thus, our new truth conditions for $>$ based on the Ramsey test will form a "conservative extension" of the old definition. However, for arbitrary $\beta \in \mathbf{L}_{B}$, especially sentences of the form $\beta=(B>C)$, we must test $B>C$ in $M_{A}^{*}$, not at $\min (M, A)$.

Definition 4 Let $M=\langle W, \leq, \varphi\rangle$ be a revision model, $A \in \mathbf{L}_{C P L}$ and $B \in \mathbf{L} . M$ satisfies $A>B$ (written $M \mid=A>B$ ) iff $M_{A}^{*} \mid=\mathrm{B} B$.

We now have a conditional connective whose truth conditions are specified directly by the Ramsey test. In particular, conditionals with arbitrary consequents have meaningful acceptance conditions; and right-nested conditionals impose constraints on the process of iterated revision. We will see, however, that their acceptance can be verified using only conditions on the original revision model.

Notice that the truth of $A>B$ is unspecified for $A \notin \mathrm{L}_{C P L}$. MC-revision functions are suitable only for sequences of objective updates. The nesting of conditionals sanctioned in a meaningful way in this framework is right-nesting, for instance, $A>(B>C)$ where $A, B, C \in \mathbf{L}_{C P L}$. A sentence $(A>B)>C$ has an unspecified truth value for it asks if $C$ is believed when a knowledge base is revised to include $A>B$. This framework does not specify how to revise a knowledge base with non-objective sentences, though we return to this problem in the concluding section.

\subsection{Minimal Change of Conditional Beliefs}

In this section, we investigate some of the properties of single changes to a revision model or belief set using the MC-revision function $*$. We assume throughout some revision model $M$ capturing the extended belief set $E$ and the belief set $K \subseteq E$. When $M$ (or $K$ or $E$ ) is revised by $A$ the properties of $K_{A}^{*}$ are obvious: $K_{A}^{*}=K_{A}^{*}$, where $*^{\mathrm{M}}$ is the AGM operator determined by $M$. Of more interest is 


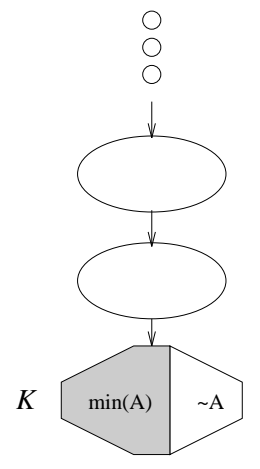

$M$

(a)

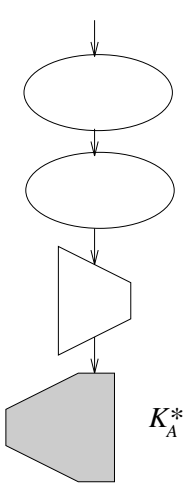

$M_{A}^{*}$

(b)

Figure 4: MC-revision with a consistent revision.

the characterization of of the new extended set $E_{A}^{*}$. Since minimal mutilation of the ordering relation $\leq$ is intended to preserve as many conditional beliefs as possible, we must determine precisely which conditionals in $E$ remain in $E_{A}^{*}$ and which are sacrificed. Since we are interested in single revisions, we restrict our attention (for the time being) to simple conditionals of the form $A>B$ where both $A$ and $B$ are objective. One important property to note is the following:

Proposition 6 Let $M$ be a $K$-revision model where $A \in K$. Then $M_{A}^{*}=M$.

Updating by a sentence already in a belief set not only causes no change in the belief set $K$, as required by the AGM postulates, but also leaves the revision model $M$ intact. More generally, consider the two scenarios, illustrated by Figures 4 and 5, that might arise when $M$ is revised by $A$. Figure 4 shows the situation where $A$ is a consistent revision, $K \forall \neg A$, while Figure 5 demonstrates the behavior of an inconsistent revision, $K \vdash \neg A$. We are interested in those simple conditionals $B>C$ that are true in the model $M_{A}^{*}$. In each of these two scenarios (in each figure) there are two cases to consider, $-B \notin K_{A}^{*}$ and $\neg B \in K_{A}^{*}$ : either $B$ is consistent with the new belief set $K_{A}^{*}$ or it is not.

Consider the first situation where $\neg B \notin K_{A}^{*}$; that is, $M \models \neg(A>\neg B)$. This means that there is some $B$-world among the set $\min (M, A)$ of minimal $A$-worlds in $M$ (the shaded region of the figures). Clearly then we have that $\min \left(M_{A}^{*}, B\right)=\min (M, A \wedge B)$; so $M_{A}^{*} \mid=B>C$ iff $M=A \wedge B>C$. Therefore, whenever $K_{A}^{*} \forall-B$, a conditional $B>C$ is in $E_{A}^{*}$ iff $A \wedge B>C$ is in $E$. Notice that the status of $B>C$ in $E$ has no bearing on its acceptance or rejection in $E_{A}^{*}$. This behavior is exactly in accordance with the AGM postulates (R7) and (R8). Any AGM revision function must ensure 


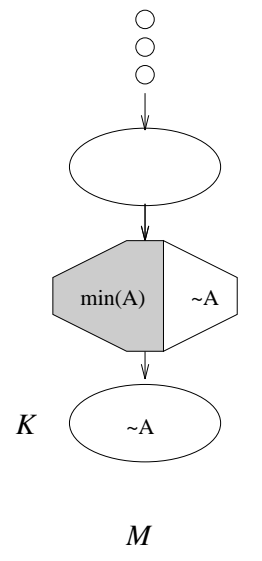

(a)

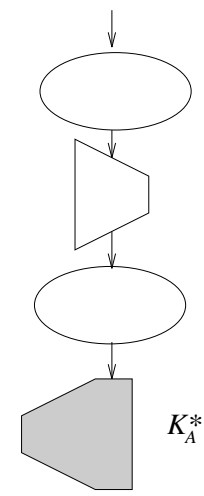

$M_{A}^{*}$

(b)

Figure 5: MC-revision with an inconsistent revision.

that subsequent (iterated) consistent revisions are treated in the same manner as uniterated consistent revisions.

The second situation arises when $\neg B \in K_{A}^{*}$; that is, $M \models A>\neg B$. When $K$ (or $E$ ) is revised by $A, \neg B$ is in the resulting belief set. This is true exactly when no $B$-world is contained in $\min (M, A)$ (again, the shaded region). Now, $M_{A}^{*} \mid=B>C$ just in case the set $\min \left(M_{A}^{*}, B\right)$ contains only $C$-worlds. Since $\neg B \in K_{A}^{*}$, the set $\min \left(M_{A}^{*}, B\right)$ is not contained in the minimal cluster of $M_{A}^{*}$. However, all worlds outside the minimal cluster stand in exactly the same relation as they do in $M$. Therefore $\min \left(M_{A}^{*}, B\right)=\min (M, B)$ and it follows that $M_{A}^{*} \mid=B>C$ iff $M \mid=B>C$. For conditionals $B>C$ whose antecedents are not made plausible by the acceptance of $A$ (i.e., $\left.K_{A}^{*} \forall \neg B\right), B>C$ is in $E_{A}^{*}$ iff $B>C$ is in $E$. Since nothing forces the conditional to be abandoned when $A$ is accepted, it is retained.

We can summarize these considerations in the following theorem and equivalent corollaries.

Theorem 7 Let $M$ be a revision model, let $*$ be the $M C$-revision operator and let $A, B, C \in \mathbf{L}_{C P L}$.

a) If $M_{A}^{*} \mid=\mathrm{B}-B$ then $M_{A}^{*} \mid=B>C$ iff $M \mid=B>C$.

b) If $M_{A}^{*} \mid \neq \mathrm{B}-B$ then $M_{A}^{*} \mid=B>C$ iff $M \mid=A \wedge B>C$.

Corollary 8 Let $M$ be a $K$-revision model and let $*$ be the $M C$-revision function.

a) If $K_{A}^{*} \vdash \neg B$ then $C \in\left(K_{A}^{*}\right)_{B}^{*}$ iff $C \in K_{B}^{*}$.

b) If $K_{A}^{*} \forall \neg B$ then $C \in\left(K_{A}^{*}\right)_{B}^{*}$ iff $C \in K_{A \wedge B}^{*}$. 
Corollary 9 Let $M$ be an E-revision model and let $*$ be the MC-revision function.

a) If $A>\neg B \in E$ then $B>C \in E_{A}^{*}$ (i.e., $A>(B>C) \in E$ ) iff $B>C \in E$.

b) If $A>\neg B \notin E$ then $B>C \in E_{A}^{*}$ (i.e., $A>(B>C) \in E$ ) iff $A \wedge B>C \in E$.

These results precisely characterize the conditionals that will be preserved in a revised extended belief set. Each shows that the sentences accepted in the new revision model or belief state can be determined by appeal to the original model or belief state. Theorem 7 shows that the conditional belief set captured by $M_{A}^{*}$ can be determined by the conditional beliefs of $M$. Furthermore, it demonstrates that $\mathrm{MC}$-revision preserves as much conditional information in the revised belief set as is consistent with the AGM postulates. The conditionals removed from $E$ when constructing $E_{A}^{*}$ are only those compelled by postulates (R7) and (R8). These are just those conditionals $B>C$ whose antecedent $B$ is consistent with $K_{A}^{*}$. This is reflected in clause b) of the theorem. However, as indicated by clause a), the remaining set of conditionals (or negated conditionals) in $E_{A}^{*}$ coincides precisely with the conditional information in the original extended set $E$. Thus, no AGM revision function could preserve more conditional information than the MC-revision function.

Corollary 8 shows that the sequence of two revisions applied to $K$ can be reduced to a single revision, requiring no iterated revision, and that the test to establish which condition holds also requires no iterated revision. Similarly, Corollary 9 shows that the revised extended belief set $E_{A}^{*}$ and the nested conditionals in $E$ can be captured by the simple, unnested conditionals in $E$. These properties will play a vital role in our characterization of revision sequences in terms of single updates.

\subsection{Revision Sequences}

The objective belief set $K_{A}^{*}$ formed by revising $K$ with $A$ relies only on one application of $*$ to $K$. Somewhat surprisingly, the simple conditionals in $E_{A}^{*}$ can also be discovered by referring only to applications of $*$ to $K$. As the results above indicate, to ask if $B>C \in E_{A}^{*}$, one first asks if $-B$ is in $K_{A}^{*}$. If this is true, then asking if $B>C \in E_{A}^{*}$ is equivalent to asking if $C \in K_{B}^{*}$. If this is false, then asking if $B>C \in E_{A}^{*}$ is equivalent to asking if $C \in K_{A \wedge B}^{*}$. A "hypothetical" revision of $K$ by $B$ or by $A \wedge B$ is sufficient to determine the status of $B>C$ in $E_{A}^{*}$ (or equivalently, the status of $A>(B>C)$ in $E)$.

Suppose we have a revision sequence, $A_{1}, \ldots A_{n}$, to be applied to $E$, where $K \subseteq E$. We can clearly use a single revision to verify whether some simple conditional $B>C$ is in $E_{A_{1}}^{*}$, or if $M_{A_{1}}^{*}=B>C$. However, if we replace $B$ by $A_{2}$ we see that these conditionals determine precisely 


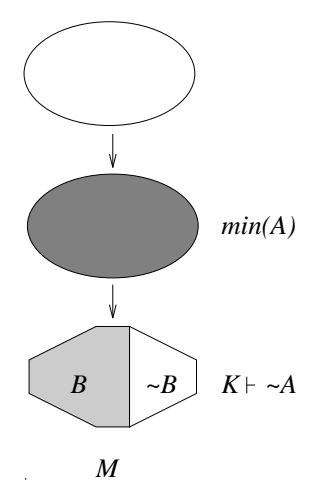

Figure 6: The order dependence of MC-revision.

the beliefs obtained when $E_{A_{1}}^{*}$ is revised by $A_{2}$, that is, $\left(E_{A_{1}}^{*}\right)_{A_{2}}^{*}$. If single revisions applied to $K$ can establish the content of the belief set obtained by an iterated revision of two levels, there seems no reason single (uniterated) revisions of $K$ cannot be used to decide the outcome of arbitrary revision sequences. We will examine the properties of such sequences and show how they may be reduced to single uniterated revisions, or unnested conditionals. This has important computational implications, for it means that queries regarding the beliefs of an agent after a sequence of revisions can be answered using only "virtual" revision of its belief set. In particular, it is not necessary to construct a new belief set and entrenchment ordering after each update. Information contained in the original ordering is sufficient to determine the result of an arbitrary sequence of revisions.

\subsubsection{Order Dependence}

The simplest true "sequence" of revisions consists of two elements $A$ and $B$. An important property of the MC-revision function applied to revision sequences is its order dependence. In general, the sets $\left(K_{A}^{*}\right)_{B}^{*}$ and $\left(K_{B}^{*}\right)_{A}^{*}$ will differ. To see this, consider a revision model $M$ such that $M \forall A>\neg B$, but $M \models B>\neg A$ (see Figure 6). When $M$ is revised by $A$, the dark shaded region of $A$-worlds become most plausible, and when $M_{A}^{*}$ is then revised with $B$, the subregion of $\min (M, A)$ containing $B$-worlds becomes most plausible. By the results above, since $-B \notin K_{A}^{*}$ we have $\left(K_{A}^{*}\right)_{B}^{*}=K_{A \wedge B}^{*}$.

In contrast, when $M$ is revised with $B$ initially, the light shaded region $\min (M, B)$ becomes most plausible, and when $M_{B}^{*}$ is revised with $A$, the dark region $\min (M, A)$ becomes most plausible, "leaving behind" the set $\min (M, B)$. Again, this is supported by our results of the previous section: since $\neg A \in K_{B}^{*}$ we have $\left(K_{B}^{*}\right)_{A}^{*}=K_{A}^{*}$. In this case, of course, $K_{A}^{*} \neq K_{A \wedge B}^{*}$, so $\left(K_{B}^{*}\right)_{A}^{*} \neq\left(K_{A}^{*}\right)_{B}^{*}$. 
For this reason, it is important to keep in mind that we are dealing with revision sequences rather than simple sets of updates. Revision of a belief or extended belief set by some set of new facts will not be order insensitive. In the example above, applying $A$ before $B$ is the same as applying $A \wedge B$ (to $K$ at least), while applying $B$ before $A$ is the same as simply revising by $A$.

This difference exists for two reasons: first, $B$ is incompatible with $A$ in the sense that $B>\neg A$ holds; second, $A$ is less plausible than $B$. Because $A$ is less plausible than $B$, revision by $A$ causes more damage to $K$ than revision by $B$. $A$ is less expected, or conflicts with $K$ to a greater degree. The belief set $K_{A}^{*}$ can be thought of as a "radical shift" in belief from $K$ (we draw a very loose analogy to Kuhn's notion of paradigm shift). When update $B$ is encountered, it must reconciled with the radically different set $K_{A}^{*}$. If it is consistent with $K_{A}^{*}$ then $\left(K_{A}^{*}\right)_{B}^{*}=K_{A \wedge B}^{*}$. In contrast, if $K$ is revised with $B$ first the results can be thought of as arising from a "routine" revision (routine in comparison to $A$ ) followed by the more "radical" revision $A$. Even though $B$ has been incorporated in $K_{B}^{*}$, the radical shift to $\left(K_{B}^{*}\right)_{A}^{*}$ offers no protection for $B$. A radical shift has little respect for most routine information in a belief set, and $B$ is as vulnerable as any other fact in $K_{B}^{*}$.

Given this interpretation, it is easy to ascertain just when the order of two revisions is irrelevant, that is, when $\left(K_{B}^{*}\right)_{A}^{*}=\left(K_{A}^{*}\right)_{B}^{*} \cdot{ }^{9}$

Definition 5 Let $M$ be a revision model for belief and extended belief sets $K$ and $E$. Updates $A$ and $B$ are mutually compatible (with respect to $M$ or $E$ ) iff $M \forall A>\neg B$ and $M \forall B>\neg A$.

Two revisions are mutually compatible just when each is a "routine" revision, relative to the other. This is equivalent to saying $A$ and $B$ are equally plausible: $A \leq_{P} B$ and $B \leq_{P} A$.

Proposition 10 If $A$ and $B$ are mutually compatible with respect to $K$-revision model $M$, then $\left(K_{B}^{*}\right)_{A}^{*}=\left(K_{A}^{*}\right)_{B}^{*}$

Now, if $A$ and $B$ are incompatible (if one is more plausible than the other) the order of revision is critical. But there are circumstances where the order may still be reversed.

Proposition 11 Let $M$ be a K-revision model such that $M \models A>\neg B$. Then $\left(K_{B}^{*}\right)_{A}^{*}=\left(K_{A}^{*}\right)_{B}^{*}$ iff $M \models B>A$.

Since $\left(K_{A}^{*}\right)_{B}^{*}=K_{B}^{*}$ whenever $A>-B$, it is easy to see that this holds. We simply observe that if

\footnotetext{
${ }^{9}$ The order of revision is "irrelevant" only with respect to the objective set $K$. Rarely will the revised models or extended sets be order insensitive. While $\left(K_{B}^{*}\right)_{A}^{*}=\left(K_{A}^{*}\right)_{B}^{*}$ may hold, it will not generally be the case that $\left(M_{B}^{*}\right)_{A}^{*}=\left(M_{A}^{*}\right)_{B}^{*}$. We will discuss this below for arbitrary sequences.
} 
$B>A$ then $K_{B}^{*}=K_{A \wedge B}^{*}$ and $\left(K_{B}^{*}\right)_{A}^{*}=K_{A \wedge B}^{*}$. Of course, the only situation left is that where $A$ and $B$ are "super-incompatible". In no instance will the order be irreversible.

Proposition 12 Let $M$ be a $K$-revision model such that $M \models A>\neg B$ and $M \models B>-A$. Then $\left(K_{A}^{*}\right)_{B}^{*}=K_{B}^{*}$, and $\left(K_{B}^{*}\right)_{A}^{*}=K_{A}^{*}$, and $K_{A}^{*} \neq K_{B}^{*}$.

\subsubsection{Reduction to Single Revisions}

In this section, we examine the possibility of simplifying the revision process. Given a revision sequence $A_{1}, \ldots A_{n}$, we would like to determine the resulting belief set $\left(\left(K_{A_{1}}^{*}\right)_{A_{2}}^{*} \ldots\right)_{A_{n}}^{*}$ without having to perform each these $n$ distinct revisions of different belief sets. In fact, we will show that any sequence of revisions can be "reduced" to a single revision. To be more precise we define a characteristic sentence for a revision sequence.

Definition 6 Let $A_{1}, \ldots A_{n}$ be a revision sequence. We say this sequence is characterized by the sentence $\alpha$ iff $\left(\left(K_{A_{1}}^{*}\right)_{A_{2}}^{*} \cdots\right)_{A_{n}}^{*}=K_{\alpha}^{*}$.

Here we show that every revision sequence has such a characteristic sentence, and that this sentence can itself be determined by the simple unnested conditionals contained in the belief set (or, using the Ramsey test, by "hypothetical" unnested revisions of $K$ ).

While mutual compatibility is sufficient to ensure that revision ordering can be reversed, we are typically more concerned with processing updates in the order they are received. When $A$ is processed before $B$, we have seen that $\left(K_{A}^{*}\right)_{B}^{*}=K_{A \wedge B}^{*}$ whenever $A>\neg B$ is false. The mutual compatibility of $A$ and $B$ is not important when revisions are processed in order. Rather the forward compatibility of $B$ with $A$ determines the content of $\left(K_{A}^{*}\right)_{B}^{*}$, and how it may be achieved with a single revision. If $B$ is forward compatible with $A$, that is, if $-B \notin K_{A}^{*}$, then $\left(K_{A}^{*}\right)_{B}^{*}$ reduces to $K_{A \wedge B}^{*}$. If $B$ is incompatible, then $\left(K_{A}^{*}\right)_{B}^{*}$ reduces to $K_{B}^{*}$. This can be extended in the obvious fashion to arbitrary sequences of revisions. ${ }^{10}$

Definition 7 Let $M$ be a $K$-revision model determining MC-revision function $*$. The revision sequence $A_{1}, \ldots A_{n}$ is forward compatible with respect to $*$ (or model $M$ ) iff $\neg A_{i+1} \notin$ $\left(\left(K_{A_{1}}^{*}\right)_{A_{2}}^{*} \cdots\right)_{A_{i}}^{*}$ for each $1 \leq i<n$.

\footnotetext{
${ }^{10} \mathrm{We}$ concentrate on revision of models and belief sets, taking for granted the straightforward connections to extended sets and nested conditionals.
} 
This can be restated as

$$
M \forall A_{1}>\left(A_{2}>\cdots\left(A_{i}>\neg A_{i+1}\right)\right)
$$

for each $i<n$. Loosely, we say that the sequence is forward compatible (or simply compatible) for $K$, when $*, M$ or $E$ is understood. Clearly, we have the following:

Proposition 13 If a revision sequence $A_{1}, \ldots A_{n}$ is forward compatible, so is each subsequence $A_{1}$, $\ldots A_{i}$ for $i \leq n$.

An obvious inductive argument, generalizing the case of the two-element sequence, gives us the following:

Theorem 14 If $A_{1}, \ldots A_{n}$ is forward compatible for $K$, then $\left(\left(K_{A_{1}}^{*}\right)_{A_{2}}^{*} \cdots\right)_{A_{n}}^{*}=K_{A_{1} \wedge \cdots A_{n}}^{*}$.

Corollary $15 A_{1}, \ldots A_{n}$ is forward compatible for $K$ iff $\neg A_{i+1} \notin K_{A_{1} \wedge \ldots A_{i}}^{*}$ for each $i<n$.

Thus (by Corollary 15) testing for compatibility can be reduced to the application of single revisions to the belief set $K$, or testing simple conditionals $A_{1} \wedge \cdots A_{i}>A_{i+1}$. Iterated revision or nested conditionals are not required to test for compatibility, nor (by Theorem 14) are they needed to compute the result of such a revision sequence applied to $K$. Computing compatible revision sequences is a straightforward extension of the case of two compatible revisions, and is reducible to a single revision, the conjunction of the elements.

In the incompatible instance, the two-element sequence was again reducible to a single revision: $\left(K_{A}^{*}\right)_{B}^{*}=K_{B}^{*}$ when $A>\neg B$. Accounting for an incompatible revision after a longer sequence of compatible revisions, however, is not so straightforward. Suppose we have a revision sequence, $A_{1}$, $\ldots A_{n+1}$, where $A_{1}, \ldots A_{n}$ is compatible but the longer sequence is not. The analogy to the twoelement case breaks down here, for in general $\left(\left(K_{A_{1}}^{*}\right)_{A_{2}}^{*} \cdots\right)_{A_{n+1}}^{*} \neq K_{A_{n+1}}^{*}$. Unfortunately, earlier revisions leave a residual trace on the structure $M$, as shown in Figure 7. While $\left(\left(K_{A_{1}}^{*}\right)_{A_{2}}^{*} \cdots\right)_{A_{n}}^{*}=$ $K_{A_{1} \wedge \cdots A_{n}}^{*}$, most certainly $\left(\left(M_{A_{1}}^{*}\right)_{A_{2}}^{*} \cdots\right)_{A_{n}}^{*}$ is different from $M_{A_{1} \wedge \cdots A_{n}}^{*}$. The history of the belief set $K$, the process by which it was formed, plays a vital role in future revisions.

In this model, the original structure of the $K$-revision model lies primarily above the dashed line. Revision by $A_{1}$ moved the minimal $A_{1}$-worlds to the cluster just below the dashed line. Since $A_{2}$ is compatible with $A_{1}$, revision by $A_{2}$ removed the $A_{2}$-worlds from this new cluster (leaving behind $A_{1} \wedge \neg A_{2}$-worlds) and moved them to the second cluster below the line. This process was repeated up to $A_{n}$, resulting in a final minimal cluster of $A_{1} \wedge \cdots A_{n}$-worlds (shaded in Figure 7). The same minimal cluster would have been formed had $K$ simply been revised by $A_{1} \wedge \cdots A_{n}$, but the sequence 


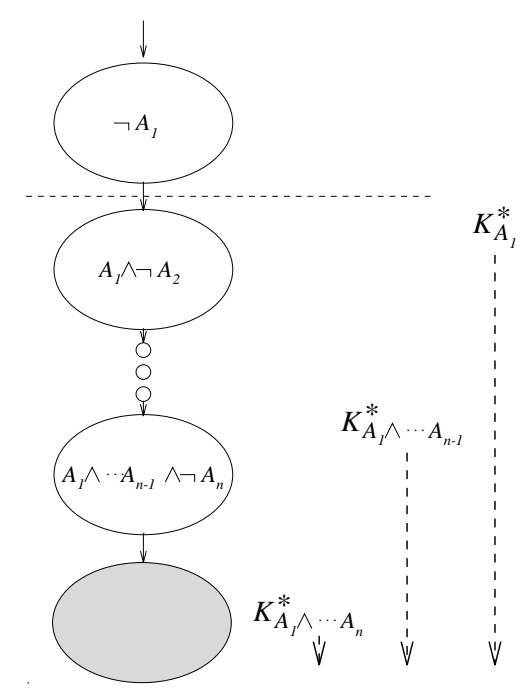

Figure 7: A sequence of compatible revisions.

of revisions has a drastically different effect on the structure of $M$, leaving a number of intermediate clusters in its wake. Simply revising by the conjunction $A_{1} \wedge \cdots A_{n}$ would have caused only the shaded cluster to form below the dashed line.

Referring still to Figure 7, if a subsequent revision $A_{n+1}$ is not compatible with the sequence $A_{1}$, $\ldots A_{n}$, then $\left(\left(K_{A_{1}}^{*}\right)_{A_{2}}^{*} \ldots\right)_{A_{n}}^{*} \vdash \neg A_{n+1}$. That is, no $A_{n+1}$-worlds can be found in the shaded minimal cluster. When $\left(\left(M_{A_{1}}^{*}\right)_{A_{2}}^{*} \cdots\right)_{A_{n}}^{*}$ is revised with $A_{n+1}$, it need not be the case that $K_{A_{n+1}}^{*}$ results. If the minimal $A_{n+1}$-worlds are found in some cluster below the dashed line, that is, if $K_{A_{1}}^{*} \forall \neg A_{n+1}$, then $\left(\left(K_{A_{1}}^{*}\right)_{A_{2}}^{*} \cdots\right)_{A_{n+1}}^{*}$ will not usually equal $K_{A_{n+1}}^{*}$.

So exactly where will minimal $A_{n+1}$-worlds be found in $\left(\left(M_{A_{1}}^{*}\right)_{A_{2}}^{*} \cdots\right)_{A_{n}}^{*}$ and what sentences will be in $\left(\left(K_{A_{1}}^{*}\right)_{A_{2}}^{*} \ldots\right)_{A_{n+1}}^{*}$ ? Suppose the sequence $A_{1}, \ldots A_{k}, A_{n+1}$ is incompatible. This means there can be no $A_{n+1}$-worlds in the cluster formed when $\left(\left(K_{A_{1}}^{*}\right)_{A_{2}}^{*} \cdots\right)_{A_{k-1}}^{*}$ is revised by $A_{k}$, that is, the cluster of $A_{1} \wedge \cdots A_{k}$-worlds. Of course, this implies that there can be no $A_{n+1}$-worlds is any lower clusters formed by the subsequent revisions $A_{k-1}$ through $A_{n}$, since each of these is compatible and will only "select" worlds from this set of $-A_{n+1}$-worlds. Conversely, if $A_{1}, \ldots A_{k}, A_{n+1}$ is a compatible sequence, there must be some $A_{n+1}$-worlds among the the cluster of $A_{1} \wedge \cdots A_{k}$-worlds representing $\left(\left(K_{A_{1}}^{*}\right)_{A_{2}}^{*} \cdots\right)_{A_{k}}^{*}$.

It now becomes clear that the minimal set of $A_{n+1}$-worlds must be located in the cluster of worlds "labeled" $A_{1} \wedge \cdots A_{k} \wedge \neg A_{k+1}$, where $k \leq n$ is maximal among the set of $i$ such that $A_{1}, \ldots A_{i}, A_{n+1}$ 
is a compatible sequence of revisions. Since $A_{1}, \ldots A_{k}$ is compatible, $\left(\left(K_{A_{1}}^{*}\right)_{A_{2}}^{*} \cdots\right)_{A_{k}}^{*}=K_{A_{1} \wedge \cdots A_{k}}^{*}$, and the minimal $A_{n+1}$-worlds in this set are captured by $\left(\left(\left(K_{A_{1}}^{*}\right)_{A_{2}}^{*} \cdots\right)_{A_{k}}^{*}\right)_{A_{n+1}}^{*}$. But this is equivalent to $K_{A_{1} \wedge \cdots A_{k} \wedge A_{n+1}}^{*}$ since $A_{n+1}$ is compatible with the rest of the sequence.

Definition 8 Let $A_{1}, \ldots A_{n}$ be a revision sequence. Update $A_{k}(k>1)$ is incompatible in this sequence iff $-A_{k} \in\left(\left(K_{A_{1}}^{*}\right)_{A_{2}}^{*} \cdots\right)_{A_{k-1}}^{*}$. A sequence is incompatible iff it contains at least one incompatible member.

Proposition 16 Sequence $A_{1}, \ldots A_{n}$ is incompatible iff it is not forward compatible.

If the belief set $\left(\left(K_{A_{1}}^{*}\right)_{A_{2}}^{*} \cdots\right)_{A_{n}}^{*}$ is characterized by a single revision $\alpha$ but $A_{n+1}$ is incompatible, then the sentence representing $\left(\left(K_{A_{1}}^{*}\right)_{A_{2}}^{*} \cdots\right)_{A_{n+1}}^{*}$ is clearly not equivalent to $\alpha \wedge A_{n+1}$. The situation we have have described above, where only the last revision in a sequence is incompatible, is easily characterized.

Theorem 17 Let $A_{1}, \ldots A_{n+1}$ be an incompatible sequence such that $A_{1}, \ldots A_{n}$ is compatible. Let $k$ be the maximal element of

$$
\left\{i \leq n: \neg A_{n+1} \notin\left(\left(K_{A_{1}}^{*}\right)_{A_{2}}^{*} \cdots\right)_{A_{i}}^{*}\right\}
$$

Then $\left(\left(K_{A_{1}}^{*}\right)_{A_{2}}^{*} \cdots\right)_{A_{n+1}}^{*}=K_{A_{1} \wedge \cdots A_{k} \wedge A_{n+1}}^{*}$.

Thus a sequence with one incompatible revision as its last element is reducible to a single revision. Notice that when the set of revisions compatible with $A_{n+1}$ is empty, when this maximal element $k$ does not exist, we have $\left(\left(K_{A_{1}}^{*}\right)_{A_{2}}^{*} \cdots\right)_{A_{n+1}}^{*}=K_{A_{n+1}}^{*}$. This is directly analogous to the two-element case, since there is no subsequence compatible with $A_{n+1}$. In Figure 7 this occurs exactly when there are no $A_{n+1}$-worlds below the dashed line; that is, when $\neg A_{n+1} \in K_{A_{1}}^{*}$.

It should be quite clear that subsequent compatible revisions, $A_{n+2}$ and so on, should be treated as previously and simply "conjoined" to $\left(\left(K_{A_{1}}^{*}\right)_{A_{2}}^{*} \cdots\right)_{A_{n+1}}^{*}$.

Proposition 18 Let $A_{1}, \ldots A_{n}$ be a revision sequence with one incompatible element $A_{k}$, and let $j<k$ be the maximal compatible revision for $A_{k}$ (as in Theorem 17). Then $\left(\left(K_{A_{1}}^{*}\right)_{A_{2}}^{*} \cdots\right)_{A_{n}}^{*}=$ $K_{A_{1} \wedge \cdots A_{j} \wedge A_{k} \wedge \cdots A_{n}}^{*}$.

The final piece in the puzzle is the process by which subsequent incompatible revisions are achieved. Consider a revision sequence $A_{1}, \ldots A_{n}$ where $A_{k}$ is incompatible and has as its most recent compatible revision $A_{j}$ (the element defined in Theorem 17). This situation is illustrated in Figure 8. Now suppose update $A_{n+1}$ is incompatible, so that no $A_{n+1}$-worlds are located in the minimal 


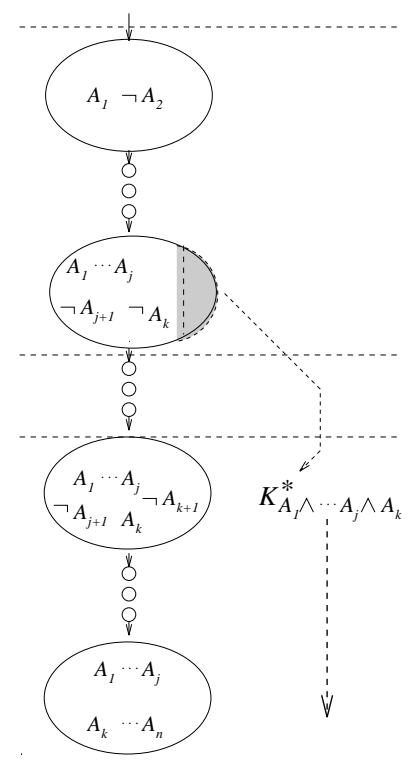

Figure 8: A sequence with an incompatible revision.

cluster. This occurs when $K_{A_{1} \wedge \cdots A_{j} \wedge A_{k} \wedge \cdots A_{n}}^{*} \vdash \neg A_{n+1}$. Again, to find the minimal $A_{n+1}$-worlds in this structure we must look for the most recent compatible revision in the sequence $A_{1}, \ldots A_{n}$. If $A_{i}$ is this update then $\left(\left(K_{A_{1}}^{*}\right)_{A_{2}}^{*} \cdots\right)_{A_{n+1}}^{*}$ is identical to $\left(\left(\left(K_{A_{1}}^{*}\right)_{A_{2}}^{*} \cdots\right)_{A_{i}}^{*}\right)_{A_{n+1}}^{*}$. Furthermore, since $A_{1}, \ldots A_{n}$ has only one incompatible revision, by Theorem 14 and Proposition 18, we have $\left(\left(K_{A_{1}}^{*}\right)_{A_{2}}^{*} \cdots\right)_{A_{i}}^{*}=K_{\alpha}^{*}$ for some sentence $\alpha$. Thus $\left(\left(K_{A_{1}}^{*}\right)_{A_{2}}^{*} \cdots\right)_{A_{n+1}}^{*}=K_{\alpha \wedge A_{i}}^{*}$. It is interesting, however, to examine the various situations that arise with respect to the occurrence of this most recent compatible revision $A_{i}$.

First, consider $i \geq k$. In this case, $A_{n+1}$ is compatible with the previous incompatible revision $A_{k}$. The set of minimal $A_{n+1}$-worlds lies below the third dashed line, among those worlds representing $K_{A_{1} \wedge \cdots A_{j} \wedge A_{k}}^{*}$. In this circumstance we have $\left(\left(K_{A_{1}}^{*}\right)_{A_{2}}^{*} \cdots\right)_{A_{n+1}}^{*}=K_{A_{1} \wedge \cdots A_{j} \wedge A_{k} \wedge \cdots A_{i} \wedge A_{n+1}}^{*}$.

Second, consider $j<i<k$. Clearly, $A_{n+1}$ is incompatible with the incompatible revision $A_{k}$, but is compatible with the sequence $A_{1}, \ldots A_{j}$. This case is rather interesting for we cannot simply "backtrack" within our representative revision for $A_{1}, \ldots A_{n}$. Because $\left(\left(K_{A_{1}}^{*}\right)_{A_{2}}^{*} \cdots\right)_{A_{n}}^{*}=$ $K_{A_{1} \wedge \cdots A_{j} \wedge A_{k} \wedge \cdots A_{n}}^{*}$, one might think we could simply "back up" to the most recent compatible revision $A_{j}$ in this representation and arrive at $K_{A_{1} \wedge \cdots A_{j}}^{*}$. However, this ignores the consistent revisions between $A_{j}$ and $A_{k}$ (between the second and third dashed lines) that were "left behind" when the incompatible revision $A_{k}$ was incorporated. The minimal $A_{n+1}$-worlds lie in this region and this 
must be taken into account. Indeed, since $A_{1}, \ldots A_{i}$ is compatible we have $\left(\left(K_{A_{1}}^{*}\right)_{A_{2}}^{*} \ldots\right)_{A_{n+1}}^{*}=$ $K_{A_{1} \wedge \cdots A_{i} \wedge A_{n+1}}^{*}$.

Finally, consider $i \leq j$. Since $\left(\left(K_{A_{1}}^{*}\right)_{A_{2}}^{*} \cdots\right)_{A_{n+1}}^{*}=K_{A_{1} \wedge \cdots A_{i}}^{*}$, this case is much like the one just mentioned. It is distinguished by the fact that the single revision $A_{1} \wedge \cdots A_{i}$ that represents it is a proper subsequence of the update $A_{1} \wedge \cdots A_{j} \wedge A_{k} \wedge \cdots A_{n}$ representing $\left(\left(K_{A_{1}}^{*}\right)_{A_{2}}^{*} \cdots\right)_{A_{n}}^{*}$.

These considerations can be generalized to accommodate any number of incompatible revisions. Furthermore, they provide a constructive means (described inductively) of reducing any sequence of revisions of belief set $K$ to a single revision of $K$, and demonstrate (through compatibility testing) how that revision can itself be determined using only single (non-iterated) revisions of $K$. Finally we shall see that, although the inductive description indicates a dependence of the characterization of $\left(\left(K_{A_{1}}^{*}\right)_{A_{2}}^{*} \cdots\right)_{A_{n}}^{*}$ on the characterization of $\left(\left(K_{A_{1}}^{*}\right)_{A_{2}}^{*} \cdots\right)_{A_{i}}^{*}$, for each $i<n$, it is only necessary to keep track of those sentences that characterize incompatible revisions.

Definition 9 Let $A_{1}, \ldots A_{n}$ be a revision sequence with $c$ incompatible updates. We use the strictly increasing function $\sigma:\{1, \cdots, c\} \mapsto\{1, \cdots, n\}$ to denote these incompatible elements: $A_{\sigma(1)}$, $\ldots A_{\sigma(c)}$. For each $1<j \leq n$, the maximal consistent incompatible revision for $A_{j}$ is $A_{k}$, where

$$
k=\max \left\{\sigma(i): \sigma(i)<j \text { and }-A_{j} \notin\left(\left(K_{A_{1}}^{*}\right)_{A_{2}}^{*} \cdots\right)_{A_{\sigma(i)}}^{*}\right\}
$$

For each $1<j \leq n$, the most recent compatible revision for $A_{j}$ is $A_{k}$, where

$$
k=\max \left\{i: i<j \text { and }-A_{j} \notin\left(\left(K_{A_{1}}^{*}\right)_{A_{2}}^{*} \cdots\right)_{A_{i}}^{*}\right\}
$$

If either of these sets is empty, we take the most recent or maximal incompatible revision for $A_{j}$ to be $\mathrm{T}$.

Lemma 19 Let $A_{1}, \ldots A_{n}$ be a revision sequence such that each proper subsequence $A_{1}, \ldots A_{i}$ is characterized by some sentence $s\left(A_{i}\right)$. Then $A_{1}, \ldots A_{n}$ is characterized by $s\left(A_{k}\right) \wedge A_{n}$, where $A_{k}$ is the most recent compatible revision for $A_{n}$. In other words, $\left(\left(K_{A_{1}}^{*}\right)_{A_{2}}^{*} \cdots\right)_{A_{n}}^{*}=K_{s\left(A_{k}\right) \wedge A_{n}}^{*}$.

This leads to the main result of this section.

Theorem 20 For any revision sequence $A_{1}, \ldots A_{n}$, there is some subset of these updates $S \subseteq$ $\left\{A_{1}, \cdots, A_{n}\right\}$ such that $\left(\left(K_{A_{1}}^{*}\right)_{A_{2}}^{*} \cdots\right)_{A_{n}}^{*}=K_{A}^{*}$ and $A=\wedge S$.

Corollary 21 For any revision sequence $A_{1}, \ldots A_{n}$, there is some subset of these updates $S \subseteq$ $\left\{A_{1}, \cdots, A_{n}\right\}$ such that $\left(\left(E_{A_{1}}^{*}\right)_{A_{2}}^{*} \cdots\right)_{A_{n-1}}^{*} \mid=A_{n}>B$ iff $E \mid=A>B$, and $A=\wedge S$. 
This result is given its constructive character by Theorem 14, but it seems to suggest that one must keep track of a characteristic sentence $s\left(A_{i}\right)$ for each revision $A_{i}$. In fact, the critical sentences are only those corresponding to incompatible revisions, $s\left(A_{\sigma(i)}\right)$. Every other characteristic sentence $s\left(A_{i}\right)$ is simply the conjunction of subsequent revisions to the most recent incompatible revision.

Theorem 22 Let $A_{1}, \ldots A_{n}$ be a revision sequence with c incompatible updates represented by $\sigma$. For each $1 \leq k \leq n$, if $A_{k}$ is a compatible revision, then $\left(\left(K_{A_{1}}^{*}\right)_{A_{2}}^{*} \cdots\right)_{A_{k}}^{*}=K_{s\left(A_{k}\right)}^{*}$, where: $\left.a\right)$ $\left.s\left(A_{k}\right)=s\left(A_{\sigma(i)}\right) \wedge A_{\sigma(i)+1} \wedge \cdots A_{k} ; b\right) \sigma(i)<k<\sigma(i+1)$, or $\sigma(i)<k$ if $i=c$; and $\left.c\right) s\left(A_{\sigma(i)}\right)$ characterizes subsequence $A_{1}, \ldots A_{\sigma(i)}$.

We can provide a similar characterization of incompatible revisions, but these must be in terms of the maximal consistent incompatible revision rather than the most recent incompatible revision.

Proposition 23 Let $A_{1}, \ldots A_{n}$ be a revision sequence with c incompatible updates represented by $\sigma$. Let $A_{\sigma(i)}$ be the maximal consistent incompatible revision for $A_{k}$. If $A_{j}$ is the most recent compatible revision for $A_{k}$, then $\sigma(i) \leq j<\sigma(i+1)$.

Theorem 24 Let $A_{1}, \ldots A_{n}$ be a revision sequence with c incompatible updates represented by $\sigma$. For each $1 \leq k \leq n$, if $A_{k}$ is an incompatible revision, then $\left(\left(K_{A_{1}}^{*}\right)_{A_{2}}^{*} \cdots\right)_{A_{k}}^{*}=K_{s\left(A_{k}\right)}^{*}$, where: a) $s\left(A_{k}\right)=s\left(A_{\sigma(i)}\right) \wedge A_{\sigma(i)+1} \wedge \cdots A_{j} \wedge A_{k}$; b) $A_{\sigma(i)}$ is the maximal incompatible revision for $A_{k}$; c) $A_{j}$ is the most recent compatible revision for $A_{k}$; and $\left.d\right) s\left(A_{\sigma(i)}\right)$ characterizes subsequence $A_{1}, \ldots A_{\sigma(i)}$.

Taken together, these theorems show that one may implement a procedure that tests for membership of $B$ in a multiply-revised belief set $\left(\left(K_{A_{1}}^{*}\right)_{A_{2}}^{*} \cdots\right)_{A_{n}}^{*}$ using only an "oracle" that answers requests of the form "Is $\beta \in K_{\alpha}^{*}$ ?" for $\alpha, \beta \in \mathrm{L}_{C P L}$. Furthermore, the characteristic sentences $s\left(A_{j}\right)$ that need to be recorded are only those of the form $s\left(A_{\sigma(i)}\right)$ where $A_{\sigma(i)}$ is some incompatible revision. The core of this algorithm is provided in Figure 9. It takes as input a revision sequence $A_{1}, \ldots A_{n}$ and computes the characteristic sentence $A$ such that $K_{A}^{*}=\left(\left(K_{A_{1}}^{*}\right)_{A_{2}}^{*} \cdots\right)_{A_{n}}^{*}$. The algorithm is incremental in the sense that a subsequent revision $A_{n+1}$ requires only one further execution of the main "for loop". To ask whether $B \in\left(\left(K_{A_{1}}^{*}\right)_{A_{2}}^{*} \cdots\right)_{A_{n}}^{*}$, one simply computes the required characteristic sentence $A$ and asks if $B \in K_{A}^{*}$.

\subsection{Information Preservation}

One desideratum of any model of the revision process is the minimization of information loss. In the AGM framework for single revisions, postulate (R4) ensures that no beliefs from set $K$ are retracted 
Input: $\quad$ revision sequence $A_{1}, \ldots A_{n}$; revision function * applicable to belief set $K$

Output: $\quad$ characteristic update $A-K_{A}^{*}=\left(\left(K_{A_{1}}^{*}\right)_{A_{2}}^{*} \cdots\right)_{A_{n}}^{*}$

Variables: $L$ - list of updates

$I L$ - list of incompatible revisions: elements have form

$\langle$ ind, $S\rangle$, where $A_{\text {ind }}$ is incompatible and subsequence

$A_{1}, \ldots A_{\text {ind }}$ is characterized by sentence $S$

$S$ - characteristic sentence $A$ built up here

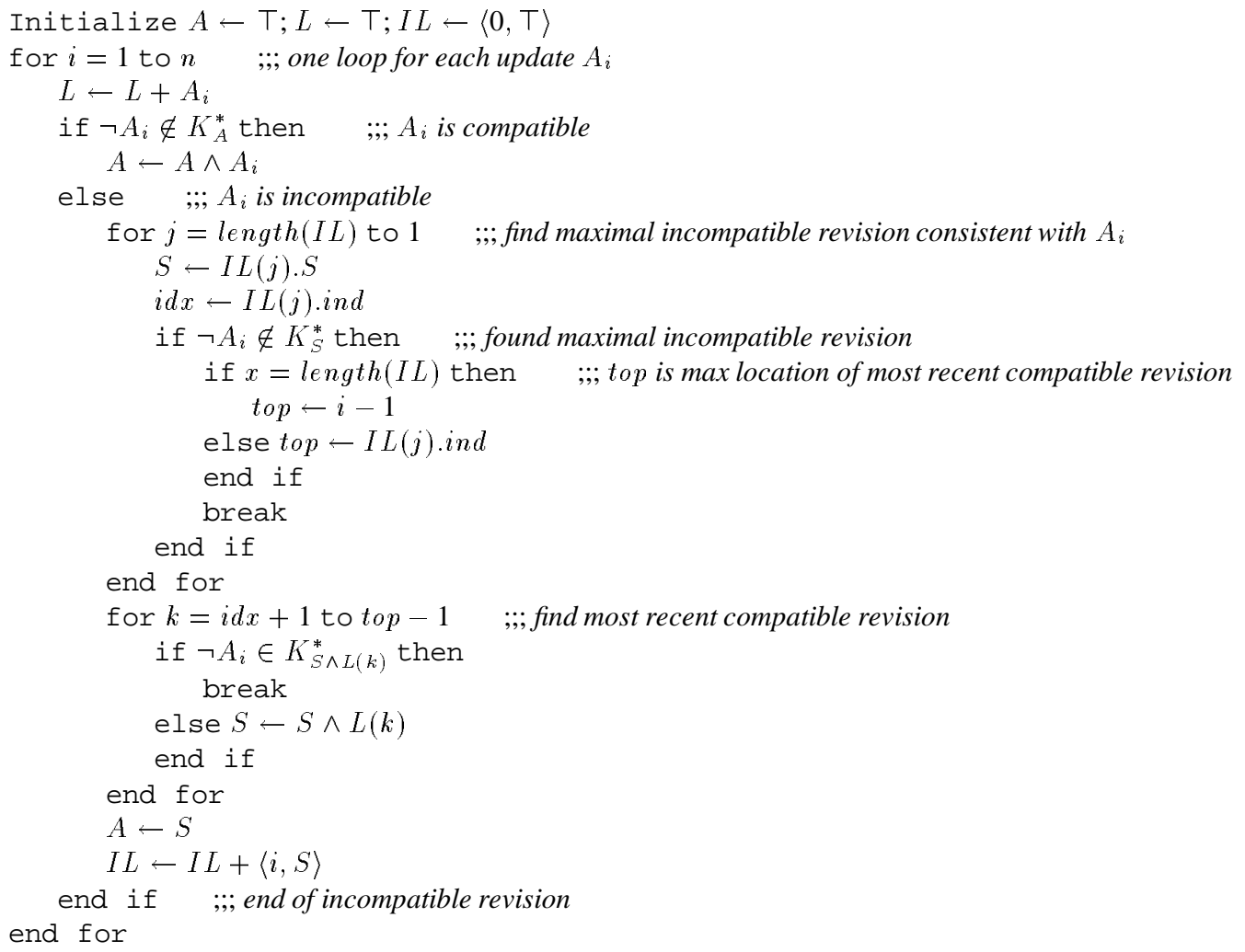

Figure 9: Algorithm to compute characteristic update $A$ for revision sequence $A_{1}, \ldots A_{n}$. 
if none need to be. An arbitrarily long sequence of consistent revisions will simply make a belief set larger, resulting (in the limit) in a complete belief set. Eventually, we will believe either $A$ or $\neg A$ for each objective sentence $A$. Once an inconsistent revision is processed, the AGM model asserts that, among those beliefs that could be given up to accommodate $A$, only those that are least entrenched are retracted. Unfortunately, no constraints are levied on the relative degrees of entrenchment of the members of the new belief set $K_{A}^{*}$. Much of the information associated with $K$, including relative degrees of entrenchment, is lost.

The MC-revision model is unique in the sense that this type of information is preserved. When a consistent revision $A$ is processed, the minimal cluster of a revision model $M$ is divided, resulting in two smaller clusters. A subsequent consistent revision breaks the minimal cluster of $M_{A}^{*}$, and so on. Eventually (supposing some finite language) after a suitable sequence of consistent revisions, we end up with a minimal cluster containing one possible world, representing a complete belief set.

The process is not altogether different for inconsistent revisions. Rather than dividing the minimal cluster of $K$-worlds, the cluster containing $\min (M, A)$ is divided. However, any information implicit in previous revisions that caused the formation of various clusters in $M$ is preserved. All other clusters remain undisturbed. In no case will a revision cause any cluster to "grow" or "lose information." Typically, a revision will cause the number of clusters to increase by one by $\operatorname{splitting} \min (M, A)$ from a cluster, thus "shrinking" a cluster and ensuring information gain. Only in certain cases will no new clusters be formed, for example, when we revise $K$ by $A$ where $A \in K$. Thus, we have a continual "thinning" of our revision models.

Proposition 25 Let revision model $M_{A}^{*}=\langle W, \leq, \varphi\rangle$ be the $M C$-revision of $M$ by $A$. If $\mathcal{C} \subseteq W$ is a cluster in $M_{A}^{*}$, then $\mathcal{C} \subseteq \mathcal{C}^{\prime}$ for some cluster $\mathcal{C}^{\prime}$ in $M$. Furthermore, if $\min (M, A) \subset \mathcal{C}^{\prime}$ for some cluster $\mathcal{C}^{\prime}$ in $M$, then the set of clusters in $M_{A}^{*}$ consists of the set of clusters in $M$ distinct from $\mathcal{C}^{\prime}$ together with $\min (M, A)$ and $\mathcal{C}^{\prime} \perp \min (M, A)$. If $\min (M, A)=\mathcal{C}^{\prime}$ for some cluster $\mathcal{C}^{\prime}$ in $M$, the the set of clusters in $M_{A}^{*}$ is identical to that in $M$.

Thus, a revision sequence $A_{1}, \ldots A_{n}$ causes a non-decreasing change in "information" in a belief state. No belief set farther along in the revision sequence can be smaller than an earlier belief set.

Corollary 26 Let $M$ be a $K$-revision model and $A_{1}, \ldots A_{n}$ a revision sequence. If $i \leq j$ then $\left(\left(K_{A_{1}}^{*}\right)_{A_{2}}^{*} \cdots\right)_{A_{j}}^{*} \not \subset\left(\left(K_{A_{1}}^{*}\right)_{A_{2}}^{*} \cdots\right)_{A_{i}}^{*}$.

This suggests that, as we process a revision sequence, our revision model becomes more and more informationally complete in the following sense. 
Definition 10 A revision model $M$ is informationally complete iff each cluster in $M$ is a singleton set.

Proposition 27 Let $M$ be an informationally complete $K$-revision model. Then $K_{A}^{*}$ is a complete theory for any $A \in \mathbf{L}_{C P L}$. Furthermore, $M_{A}^{*}$ is informationally complete.

Corollary 28 If $M$ is an informationally complete $K$-revision model then $K$ is a complete theory.

Proposition 25 ensures furthermore that $M_{A}^{*}$ will be informationally complete whenever $M$ is. It also shows that if we restrict our attention to languages with a finite number of propositional variables, we can eventually attain informational completeness. ${ }^{11}$

Proposition 29 Let $M$ be a revision model and $A_{1}, \ldots A_{n}$ a revision sequence such that every satisfiable sentence $\alpha$ is represented in this sequence; that is, $A_{i} \vdash \alpha$ for some $A_{i}$. Then $\left(\left(M_{A_{1}}^{*}\right)_{A_{2}}^{*} \cdots\right)_{A_{n}}^{*}$ is informationally complete.

An example of such a revision sequence, for a language with $p$ propositional variables, would be the set of the $2^{p}$ complete (truth-functionally distinct) conjunctions of literals, each capturing a possible world or valuation, and each causing the corresponding world to be broken off into its own cluster. From that point on all revisions will result in complete belief sets since the set of most plausible $A$-worlds will have one element for each revision $A$.

\section{Reasoning with Revision Sequences}

The algorithm for reducing nested conditionals requires some method of establishing the truth of $B \in K_{A}^{*}$ for $A, B \in \mathbf{L}_{C P L}$. Typically, as is the case in most reasoning tasks, our premises do not provide us with complete knowledge, and we can only hope to derive as much as possible, leaving certain gaps in our knowledge. It is not reasonable to expect a completely specified revision function *, or equivalently, a complete set of conditionals containing one of $A>B$ or $\neg(A>B)$, among our set of premises. Naturally, the algorithm can then easily be modified to ask whether $A>B$ or $-(A>B)$ is provable from a given premise set. ${ }^{12}$ If either is the case, the algorithm can proceed, having an answer to the query $B \in K_{A}^{*}$. If neither is derivable from the premises, then the algorithm

\footnotetext{
${ }^{11}$ We assume that models contain no "duplicate" worlds having the same associated valuation. If so, our informational completeness in the following result is the type that ensures that $K_{A}^{*}$ is complete for all $A$.

${ }^{12}$ This requires a logic in which conditionals can be expressed and reasoned with, e.g., [9].
} 
must halt unsuccessfully, or proceed as if either could be the case. ${ }^{13}$ As should be expected when reasoning with incomplete information, the answer "unknown" must be returned for certain queries.

While the revision function $*$ is usually specified only partially by means of conditional premises (as well as direct statements of plausibility and entrenchment), there may be circumstances when the revision function is completely known. The problem changes from that of reasoning with incomplete information to that of specifying complete information in a reasonable manner. We cannot expect one to specify a complete conditional theory explicitly containing $A>B$ or $\neg(A>B)$ for each objective $A$ and $B$, for we do not want to be forced to reason with infinite sets of premises. Even for finite languages with $p$ atomic variables, where we require only a conditional or its negation for semantically distinct $A$ and $B$, we are forced to reason with $2^{p+1}$ premises. ${ }^{14}$

There are cases, though, where a revision function can be captured finitely, and often with a manageable number of sentences. Often when a set of conditional premises is given, we have in mind a certain intended model (e.g., preferred entrenchment orderings are described in [26, 4, 28]), and these can often be represented finitely [4]. In general, a revision function can be compactly represented if there is a corresponding revision model that is "well-behaved" in the following sense.

Definition 11 A revision model $M=\langle W, \leq, \varphi\rangle$ is finitely specifiable iff $W=\cup_{i \leq n} \mathcal{C}_{i}$, where each $\mathcal{C}_{i}$ is a cluster in $M$ and $\mathcal{C}_{i}=\left\|S_{i}\right\|$ for some sentence $S_{i} \in \mathbf{L}_{C P L}$.

In other words, $M$ is well-behaved if it consists of a finite number of clusters, each corresponding to some finite classical theory or sentence. It is easy to verify that if $M$ is finitely specified by sentences $S_{1}, \ldots S_{n}$ in the definition above, then these sentences are "mutually exclusive" and "exhaustive"; that is, $\vdash S_{i} \supset-S_{j}$ if $i \neq j$, and $\vdash S_{1} \vee \cdots S_{n}$. We assume that $S_{1}$ characterizes the minimal (most plausible) cluster of $M, S_{2}$ the next most plausible and so on. We denote the model described above by $F S M\left(S_{1}, \cdots S_{n}\right) \cdot{ }^{15}$

Definition 12 A revision function $*$ is finitely specifiable (for belief set $K$ ) iff there is some finitely specifiable $K$-revision model $M=F S M\left(S_{1}, \cdots S_{n}\right)$ such that $*^{\mathrm{M}}=*$.

Given a finitely specifiable revision function for belief set $K$ (in which case $K$ is also finitely

\footnotetext{
${ }^{13}$ It often makes sense to continue, for a subsequent revision may be incompatible with previous revisions, and failing to reduce an earlier revision may have no effect on the effort to reduce an entire sequence.

${ }^{14}$ Alternatively, one could provide a complete set of entrenchment sentences, specifying the relative degrees of entrenchment of each pair of sentences: $A \leq_{E} B$ and/or $B \leq_{E} A$ for each $A, B \in \mathrm{L}_{C P L}$. This would allow the derivation of every simple conditional or its negation, and seems to be what Gärdenfors and Makinson [17, 25] have in mind in their presentation of expectation inference (see also [16]).

${ }^{15}$ Such a model can be described compactly in the logic $\mathrm{CO}^{*}$ as a function of the sentences $S_{1}, \cdots S_{n}$ [6].
} 
specified by $S_{1}$ ), we can use the sequence of sentences $S_{1}, \cdots S_{n}$ to represent the model $M$, as well as determine the truth of every simple conditional sentence.

Theorem 30 Let $S_{1}, \cdots S_{n} \in \mathbf{L}_{C P L}$ be such that $\vdash S_{i} \supset \neg S_{j}$ if $i \neq j$, and $\vdash S_{1} \vee \cdots S_{n}$. For all $A, B \in \mathbf{L}_{C P L}$ either

$$
\begin{aligned}
& \operatorname{FSM}\left(S_{1}, \cdots S_{n}\right)=A>B ; \quad \text { or } \\
& \operatorname{FSM}\left(S_{1}, \cdots S_{n}\right)=-(A>B)
\end{aligned}
$$

Fortunately, the ability to finitely specify a revision model is not disturbed by MC-revision. If $\operatorname{FS} M\left(S_{1}, \cdots S_{n}\right)$ characterizes a revision model $M$, then $M_{A}^{*}$ is formed by simply "dividing" the minimal cluster consistent with $A$ in two clusters: $S_{i} \wedge A$ becomes most plausible; and $S_{i} \wedge \neg A$ replaces cluster $S_{i}$.

Theorem 31 Let revision model $M=F S M\left(S_{1}, \cdots S_{n}\right)$ and let $S_{k}$ be the minimal sentence in this set consistent with $A$; that is, $S_{k} \nvdash \neg A$ and $S_{i} \vdash \neg A$ if $i<k$. Then $M_{A}^{*}$ is characterized by

$$
\begin{array}{ll}
F S M\left(S_{k} \wedge A, S_{1}, \cdots S_{k-1}, S_{k} \wedge \neg A, S_{k+1}, \cdots S_{n}\right) & \text { if } S_{k} \nvdash A \\
F S M\left(S_{k}, S_{1}, \cdots S_{k-1}, S_{k+1}, \cdots S_{n}\right) & \text { if } S_{k} \vdash A
\end{array}
$$

Thus we can completely specify a revision function with a compact set of premises and use this premise set to reduce nested conditional queries to simple conditionals and then establish the truth of these simple conditionals. Furthermore, we can explicitly revise a model and retain a compact representation. It also becomes clear that testing for the truth of a simple conditional in such a revision model is reducible to a simple propositional reasoning task.

Relative to propositional satisfiability tests, this "algorithm" is relatively efficient, for the minimal $A$-consistent cluster or sentence can be found using a linear search technique. Thus we need only perform $O(n)$ satisfiability tests to determine the truth of $A>B$. The complexity of these tests will depend on the size of the theories $S_{i}$ and their structure. For instance, if the $S_{i}$ are Horn theories, this test will be linear in the size of the theory.

\section{Concluding Remarks}

We have presented a model for iterated revision that captures sequences of objective revisions. The hallmark of MC-revision is the preservation of subjective information, such as conditional beliefs and entrenchment, throughout such a sequence. Because this information is retained across belief sets, simply knowing how a revision function behaves on single revisions is sufficient to characterize the 
results of any sequence of revisions. If we adopt the Ramsey test for acceptance of conditionals, this demonstrates that right-nested conditionals are equivalent to simple unnested conditionals, and that this reduction can be performed using only knowledge of unnested conditionals.

The MC-revision model has other compelling properties as well. Because it preserves conditional information, the sequence of belief sets corresponding to a revision sequence can never decrease in propositional information content. It preserves nice properties of the revision models and functions being revised as well, for example, informational completeness and finite specifiability. In cases where a revision function is described simply (e.g., by a set of propositional sentences), the characterization of MC-revision is also easily computed.

\subsection{Fixed Sentence Orderings}

Several other proposals have been put forth that extend the AGM theory (or variants of it) to deal with iterated revision, but these all have a somewhat different nature. In particular, the question of minimizing the change in the conditional beliefs of an agent appears to have been unaddressed. Safe contraction [3], generalized epistemic entrenchment [29] and the probabilistically motivated system of Schlechta [30] each take a similar approach to the problem: each assumes the existence of a "global" ordering of entrenchment over all sentences in the language. For any belief set $K$ the appropriate revision function is immediately available, and iteration of the process requires no additional apparatus. These models have the rather severe drawback that any objective belief set $K$ is associated with a unique revision function. In particular, $K$ uniquely determines the set of conditional beliefs. So while the ordering of entrenchment can change as $K$ evolves, these changes are predetermined by the global ordering; the entrenchment ordering associated with $K$ cannot depend on how $K$ evolved. This stands in contrast with our model, where the ordering itself can be revised. Hansson [21] makes a similar observation, that many systems of iterated change fix the connection between objective and extended sets. Hansson proposes that instead a revision method be associated with belief bases rather belief sets. Thus, the same belief set may be revised in different ways if it is generated by different bases in each instance. While clearly the issue of base revision is crucial, it is not a problem we address here. In particular, the revision of conditional beliefs depends on existing conditional beliefs and, as in the AGM theory, there need not be strong ties to the underlying belief base. $^{16}$

\footnotetext{
${ }^{16}$ Of course, such ties can be added. Work on base contraction $[20,21,13]$ can be viewed in this light.
} 


\subsection{Nonminimal Change of Conditionals}

The proposal most similar in structure and spirit to ours is that of Spohn [31], and indeed inspired this investigation of MC-revision. Spohn's use of ordinal conditional functions to represent belief states is much like the possible worlds model we have used, except that "clusters" are given explicit ordinal rankings. Spohn's notion of conditionalization on such models assigns to a proposition $A$ a new "degree of belief" or ordinal ranking (which should be thought of as the degree of entrenchment). This is analogous to revising by $A$, and is achieved by "shifting" the rank of all $A$-worlds so that the minimal $A$-worlds have an ordinal ranking that is lower (or more plausible) than that of any $-A$-world by the specified degree of belief. The key distinction between Spohn's approach and ours is that MC-revision by $A$ requires the shift of only the minimal $A$-worlds. Spohn's approach is more reminiscent of probabilistic conditionalization, and if we applied our truth conditions for conditionals to OCF update, we would see a much larger change in conditional beliefs in general.

Spohn's approach is certainly more sophisticated, and in many ways is more compelling than MC-revision. In particular, when evidence $A$ is brought to light all $A$-worlds become somewhat more plausible. However, this $\kappa$-conditionalization cannot be applied in our setting, for it requires that degrees of entrenchment be given an explicit quantitative interpretation and that the strength of the evidence be quantified on the same scale. ${ }^{17}$ MC-revision is an applicable in settings where only qualitative entrenchment information is available. Indeed, it is hard to imagine what possible shifts of nonminimal $A$-worlds are justifiable if only the relative plausibility of worlds is known. Furthermore, the MC-model requires the simple shifting of one partial cluster, a more computationally feasible mechanism for revising a ranking than shifting all $A$-worlds. Thus, MC-revision may prove to be a useful approximation method in quantitative settings as well. The utility of MC-revision is especially noticeable if new evidence tends not to contradict earlier evidence.

Darwiche and Pearl [12] propose two postulates extending the eight AGM postulates that govern iterated revision. These impose rather mild constraints on the conditionals (or ordering) that results from a revision; indeed, both Spohn's OCF model (or more accurately a suitable qualitative abstraction thereof) and MC-revision satisfy these postulates. Hence, their proposal cannot be used to fix a particular revision method (as the AGM postulates cannot). What is missing, and what will allow the exploration and specification of iterated revision methods, is a means to express the relative entrenchment of conditional sentences. This has received little attention in the literature, but several possibilities exist (e.g., using nested conditional sentences as in Section 4 without the specific

\footnotetext{
${ }^{17}$ Spohn's use of OCFs requires that the magnitudes of the rankings are meaningful.
} 
constraints of MC-revision; see also [10,11]). With this in place, a general theory such as that of Darwiche and Pearl can be used to construct revision functions.

Darwiche and Pearl also suggest that the constraints of MC-revision are too strong, for they give little "priority" to incoming evidence. An example of this is the following: we believe that Mary won't come to the party, $\bar{M}$, and that Ted won't either, $\bar{T}$. We necessarily believe the rule $\bar{M} \Rightarrow \bar{T}$. But Ted may or may not come should Mary decide to. We are first told that $M$ (and now are uncommitted to $T$ or $\bar{T}$ ) and then that $T$. Some time later we are informed that we were correct initially and Mary isn't coming $\bar{M}$ after all. Should we still believe that Ted is coming? This depends on the relative importance or weight of rules versus plain beliefs. If we accept the rule $\bar{M} \Rightarrow \bar{T}$ more strongly than the evidence $T$, then we should return to our original state of belief $(\bar{M}, \bar{T})$ as MC-revision suggests. This is certainly plausible given this scenario. However, if priority is to be given to the evidential report, then perhaps we should abandon our rule. One way MC-revision can be interpreted is as a system in which ultimate priority is given to existing rules. Darwiche and Pearl seem to come down on the side of evidential superiority (and argue that one should keep believing $T$ ) - a view that is just as extreme. Again, a general theory for specifying the relative importance of conditionals and propositional beliefs will allow one to adopt less extreme revision methods. With such a theory in place, MC-revision can still be viewed as a computationally attractive approximation method for such "ideal" revision. ${ }^{18}$

\subsection{Nested Conditionals}

It is also interesting to note that a nested conditional $A>(B>C)$ is often equivalent to $A \wedge B>C$ (whenever $A$ and $B$ are compatible). It has been suggested by a number of people that nested conditionals should be reduced to unnested conditionals with all antecedents conjoined to form a single antecedent, Adams [1] and Levi [23] among them. This reduction is sanctioned by the MCrevision model for nested conditionals with compatible antecedents. But as described in Section 4, this is not the case when incompatible antecedents are present. For instance, $A>(B>C)$ reduces simply to $B>C$ when $A$ and $B$ are incompatible. As suggested by Levi, this reduction may seem inappropriate in normal linguistic usage, for the nested conditional seems to imply that $A$ should continue to hold when $B>C$ is evaluated with the Ramsey test, thus suggesting the reduction to $A \wedge B>C$. Indeed, the MC-revision model cannot account for this circumstance when incompatible

\footnotetext{
${ }^{18}$ The party example is a rewording of the example in [12] of observing a red bird. That example is somewhat misleading for it describes an instance of adding new beliefs (about new domain objects) rather than revising old beliefs.
} 
antecedents are involved. In contrast, our model provides non-trivial acceptance conditions for nested conditionals such as $A>(-A>B)$.

Levi [23] is rather critical of the enterprise of determining truth or acceptance conditions for nested conditionals, or even allowing conditionals to be part of a belief set. He offers the opinion that an element of a belief set or corpus ought to be practicable as a standard of serious possibility. Since conditionals do not perform this function, they are not accorded the status of beliefs. While certainly their role differs from that of garden-variety propositional beliefs, they perform an indispensable function in the process of deliberation. Conditionals suggest hypothetical possibilities to an agent and aid an agent in changing its mind. If conditionals are not "beliefs," they at least must be representations of an agent's revision policies; and such policies themselves must be the objects of revision from time to time. Whether or not such policies are called beliefs, a model of conditional revision is crucial.

A few words on triviality are in order, as well. The Gärdenfors triviality result ensures that no meaningful notion of belief revision can be applied to belief sets that contain conditionals in accordance with the Ramsey test and satisfy all eight AGM postulates. ${ }^{19}$ It is clear that MC-revision is a revision function that can be applied to conditional belief sets in a nontrivial fashion, giving rise to new conditional belief sets. ${ }^{20}$ Furthermore, by Proposition 5, MC-revision applied to $K$ satisfies all AGM postulates. Notice, however, that triviality is avoided because the MC-revision function satisfies the postulates when restricted to $K$. Thus, the postulates do hold when we consider the "objective component" of the revision, but not when considered with respect to an extended set $E$. Upon reflection, however, it becomes clear that the postulates, in particular postulates pertaining to consistent revisions such as (R4), lose much of their appeal when applied to conditional belief sets. As argued convincingly by Levi [23] and Rott [27], among others, the proper escape from "triviality" requires relaxing the requirements of the AGM theory when discussing non-objective belief sets.

\subsection{Extensions}

There are a number of interesting avenues that remain to be explored. This model is restricted to rightnested conditionals, or propositional revisions. In general, we want to allow revision of a knowledge base with conditional information, or statements of entrenchment as well. A fully general model of this type is currently under investigation. Some preliminary results may be found in $[10,11]$. Other models that allow arbitrary nesting include Hansson's [21] base-set model.

\footnotetext{
${ }^{19}$ Indeed, only a few of the properties of AGM operators are required for this result.

${ }^{20}$ One need only construct a $K$-revision model for a suitably rich $K$ to show that MC-revision is nontrivial.
} 
We are currently developing a model of revision and conditionalization for single revisions that adds to the basic revision model probabilistic degrees of belief [7]. If this model can be grafted onto the MC-revision model, "conditional objects" that make statements of conditional probability can be nested in a meaningful way, and given a natural semantics. We are also exploring the application of these ideas to the processes of $J$-conditionalization and L-conditionalization. These models were proposed by Goldszmidt and Pearl [18] to capture changes in belief that have degrees of certainty attached, these degrees corresponding to Spohn's OCFs. Both extensions of MC-revision (either probabilistic or possibilistic) offer ways of attaching quantitative strength measurements to our conditionals and revising these conditionals.

Finally, this model reflects the bias of the AGM model to accepting without question the most recent update. The primacy of the most recent information is clearly not a principle that should be accepted in all circumstances. Sometimes things we learn are so radically incompatible with our knowledge that we reject them out of hand, and do not attempt to reconcile them with our current beliefs. Generalizing the AGM and MC-revision models in this way is a difficult task, but one that certainly deserves inquiry.

\section{Acknowledgements}

Thanks to Verónica Becher, Moisés Goldszmidt and Mirek Truszczyński for helpful discussions. An anonymous referee provided some very helpful suggestions and pointers to relevant literature. This research was supported by NSERC Research Grant OGP0121843.

\section{References}

[1] Ernest W. Adams. The Logic of Conditionals. D.Reidel, Dordrecht, 1975.

[2] Carlos Alchourrón, Peter Gärdenfors, and David Makinson. On the logic of theory change: Partial meet contraction and revision functions. Journal of Symbolic Logic, 50:510-530, 1985.

[3] Carlos Alchourrón and David Makinson. On the logic of theory change: Safe contraction. Studia Logica, 44(4):405-422, 1985.

[4] Craig Boutilier. Inaccessible worlds and irrelevance: Preliminary report. In Proceedings of the Twelfth International Joint Conference on Artificial Intelligence, pages 413-418, Sydney, 1991.

[5] Craig Boutilier. Epistemic entrenchment in autoepistemic logic. Fundamenta Informaticae, 17(1-2):5-30, 1992.

[6] Craig Boutilier. Sequences of revisions: On the semantics of nested conditionals. Technical Report 92-24, University of British Columbia, Vancouver, 1992. 
[7] Craig Boutilier. The probability of a possibility: Adding uncertainty to default rules. In Proceedings of the Ninth Conference on Uncertainty in AI, pages 461-468, Washington, D.C., 1993.

[8] Craig Boutilier. Conditional logics of normality: A modal approach. Artificial Intelligence, 68:87-154, 1994.

[9] Craig Boutilier. Unifying default reasoning and belief revision in a modal framework. Artificial Intelligence, 68:33-85, 1994.

[10] Craig Boutilier and Moisés Goldszmidt. Revision by conditional beliefs. In Proceedings of the Eleventh National Conference on Artificial Intelligence, pages 649-654, Washington, D.C., 1993.

[11] Craig Boutilier and Moisés Goldszmidt. A theory of conditional belief revision. In G. Crocco, L. Fari nas del Cerro, and A. Herzig, editors, Conditional Logics in Artificial Intelligence. Oxford University Press, London, 1994. (to appear).

[12] Adnan Darwiche and Judea Pearl. On the logic of iterated belief revision. In Proceedings of the Fifth Conference on Theoretical Aspects of Reasoning about Knowledge, pages 5-23, Pacific Grove, 1994.

[13] André Fuhrmann. Theory contraction through base contraction. Journal of Philosophical Logic, 20:175203, 1991.

[14] Peter Gärdenfors. Belief revisions and the Ramsey test for conditionals. The Philosophical Review, 95:81-93, 1986.

[15] Peter Gärdenfors. Knowledge in Flux: Modeling the Dynamics of Epistemic States. MIT Press, Cambridge, 1988.

[16] Peter Gärdenfors and David Makinson. Revisions of knowledge systems using epistemic entrenchment. In Proceedings of the Third Conference on Theoretical Aspects of Reasoning about Knowledge, pages 83-95, Pacific Grove, 1988.

[17] Peter Gärdenfors and David Makinson. Nonmonotonic inference based on expectations. Artificial Intelligence, 65:197-245, 1994.

[18] Moisés Goldszmidt and Judea Pearl. Reasoning with qualitative probabilities can be tractable. In Proceedings of the Eighth Conference on Uncertainty in AI, pages 112-120, Stanford, 1992.

[19] Adam Grove. Two modellings for theory change. Journal of Philosophical Logic, 17:157-170, 1988.

[20] Sven Ove Hansson. In defense of base contraction. Synthese, 91:239-245, 1992.

[21] Sven Ove Hansson. In defense of the Ramsey test. Journal of Philosophy, 89:522-540, 1992.

[22] Hector J. Levesque. All I know: A study in autoepistemic logic. Artificial Intelligence, 42:263-309, 1990.

[23] Isaac Levi. Iteration of conditionals and the Ramsey test. Synthese, 76(1):49-81, 1988.

[24] David Lewis. Counterfactuals. Blackwell, Oxford, 1973.

[25] David Makinson and Peter Gärdenfors. Relations between the logic of theory change and nonmonotonic logic. In Andre Fuhrmann and Michael Morreau, editors, The Logic of Theory Change, pages 185-205. Springer-Verlag, Berlin, 1990.

[26] Judea Pearl. System Z: A natural ordering of defaults with tractable applications to default reasoning. In M. Vardi, editor, Proceedings of Theoretical Aspects of Reasoning about Knowledge, pages 121-135. Morgan Kaufmann, San Mateo, 1990. 
[27] Hans Rott. Conditionals and theory change: Revisions, expansions, and additions. Synthese, 81(1):91-113, 1989.

[28] Hans Rott. A nonmonotonic conditional logic for belief revision. In A. Fuhrmann and M. Morreau, editors, The Logic of Theory Change, pages 135-181. Springer-Verlag, Berlin, 1990.

[29] Hans Rott. Belief change using generalized epistemic entrenchment. Journal of Logic, Language and Information, 1(1):45-78, 1992.

[30] Karl Schlechta. Theory revision and probability. Notre Dame Journal of Formal Logic, 307(2):307-318, 1991.

[31] Wolfgang Spohn. Ordinal conditional functions: A dynamic theory of epistemic states. In W.L. Harper and B. Skyrms, editors, Causation in Decision, Belief Change and Statistics, volume 2, pages 105-134. D. Reidel, Dordrecht, 1987.

[32] Robert C. Stalnaker. A theory of conditionals. In W.L. Harper, R. Stalnaker, and G. Pearce, editors, Ifs, pages 41-55. D. Reidel, Dordrecht, 1968. 1981.

[33] Robert C. Stalnaker. Inquiry. MIT Press, Cambridge, 1984.

\section{A Proofs of Main Results}

The truth of many of the propositions in the paper is rather obvious and their proofs are excluded. Certain results are described in the body of the paper and have their proofs sketched there. These proofs are also excluded.

Theorem 7 Let $M$ be a revision model, let $*$ be the $M C$-revision operator and let $A, B, C \in \mathbf{L}_{C P L}$.

a) If $M_{A}^{*} \mid=\mathrm{B}-B$ then $M_{A}^{*} \mid=B>C$ iff $M \mid=B>C$.

b) If $M_{A}^{*} \mid \neq \mathrm{B}-B$ then $M_{A}^{*} \mid=B>C$ iff $M \mid=A \wedge B>C$.

Proof The proof of this theorem is sketched, for the most part, in the text preceding its statement in the body of the paper.

Theorem 17 Let $A_{1}, \ldots A_{n+1}$ be an incompatible sequence such that $A_{1}, \ldots A_{n}$ is compatible. Let $k$ be the maximal element of

$$
\left\{i \leq n: \neg A_{n+1} \notin\left(\left(K_{A_{1}}^{*}\right)_{A_{2}}^{*} \cdots\right)_{A_{i}}^{*}\right\}
$$

Then $\left(\left(K_{A_{1}}^{*}\right)_{A_{2}}^{*} \cdots\right)_{A_{n+1}}^{*}=K_{A_{1} \wedge \cdots A_{k} \wedge A_{n+1}}^{*}$.

Proof Let $M$ be the revision model for $K$ and $*$ the MC-revision function. Let $A_{k}$ be the last element of $A_{1}, \ldots A_{n+1}$ such that $-A_{n+1} \notin\left(\left(K_{A_{1}}^{*}\right)_{A_{2}}^{*} \cdots\right)_{A_{k}}^{*}$. 
a) If there is no such element then $K_{A_{1}}^{*} \vdash \neg A_{n+1}$. The minimal cluster of $M_{A_{1}}^{*}$ is formed by the set $\min \left(M, A_{1}\right)$. Since the relative ordering of all other worlds is unaffected by this revision, $\min \left(M_{A_{1}}^{*}, A_{n+1}\right)=\min \left(M, A_{n+1}\right)$. Now, each subsequent revision is compatible, so $\min \left(\left(\left(M_{A_{1}}^{*}\right)_{A_{2}}^{*} \cdots\right)_{A_{i-1}}^{*}, A_{i}\right)$ is contained in the minimal cluster of $\left(\left(M_{A_{1}}^{*}\right)_{A_{2}}^{*} \cdots\right)_{A_{i-1}}^{*}$ for each $i \leq n$. An obvious inductive argument shows that (since no $A_{n+1}$-worlds are contained in the first minimal cluster)

$$
\min \left(\left(\left(M_{A_{1}}^{*}\right)_{A_{2}}^{*} \cdots\right)_{A_{n}}^{*}, A_{n+1}\right)=\min \left(M, A_{n+1}\right)
$$

Hence $\left(\left(K_{A_{1}}^{*}\right)_{A_{2}}^{*} \cdots\right)_{A_{n+1}}^{*}=K_{A_{n+1}}^{*}$.

b) If such a $k$ exists the, by Proposition 13 and Theorem 14,

$$
\left(\left(K_{A_{1}}^{*}\right)_{A_{2}}^{*} \cdots\right)_{A_{k}}^{*}=K_{A_{1} \wedge \cdots A_{k}}^{*}
$$

Furthermore, since $\left(\left(K_{A_{1}}^{*}\right)_{A_{2}}^{*} \cdots\right)_{A_{k}}^{*} \forall \neg A_{n+1}$, the set $\min \left(\left(\left(M_{A_{1}}^{*}\right)_{A_{2}}^{*} \cdots\right)_{A_{k}}^{*}, A_{n+1}\right)$ lies within the minimal cluster of $\left(\left(M_{A_{1}}^{*}\right)_{A_{2}}^{*} \cdots\right)_{A_{k}}^{*}$. Since sequence $A_{1}, \ldots A_{n}$ is compatible, $\min \left(\left(\left(M_{A_{1}}^{*}\right)_{A_{2}}^{*} \cdots\right)_{A_{k}}^{*}, A_{k+1}\right)$ must also lie within the minimal cluster of $\left(\left(M_{A_{1}}^{*}\right)_{A_{2}}^{*} \cdots\right)_{A_{k}}^{*}$. However, by the maximality of $k$, this set must be disjoint from the set $\min \left(\left(\left(M_{A_{1}}^{*}\right)_{A_{2}}^{*} \cdots\right)_{A_{k}}^{*}, A_{n+1}\right)$. Thus,

$$
\min \left(\left(\left(M_{A_{1}}^{*}\right)_{A_{2}}^{*} \cdots\right)_{A_{k+1}}^{*}, A_{n+1}\right)=\min \left(\left(\left(M_{A_{1}}^{*}\right)_{A_{2}}^{*} \cdots\right)_{A_{k}}^{*}, A_{n+1}\right)
$$

Since all subsequent revisions up to $A_{n+1}$ are compatible, as in case a) above,

$$
\min \left(\left(\left(M_{A_{1}}^{*}\right)_{A_{2}}^{*} \cdots\right)_{A_{n}}^{*}, A_{n+1}\right)=\min \left(\left(\left(M_{A_{1}}^{*}\right)_{A_{2}}^{*} \cdots\right)_{A_{k}}^{*}, A_{n+1}\right)
$$

But $A_{n+1}$ is compatible with $A_{1}, \ldots A_{k}$, so

$$
\left(\left(K_{A_{1}}^{*}\right)_{A_{2}}^{*} \cdots\right)_{A_{n+1}}^{*}=K_{A_{1} \wedge \cdots A_{k} \wedge A_{n+1}}^{*}
$$

Lemma 19 Let $A_{1}, \ldots A_{n}$ be a revision sequence such that each proper subsequence $A_{1}, \ldots A_{i}$ is characterized by some sentence $s\left(A_{i}\right)$. Then $A_{1}, \ldots A_{n}$ is characterized by $s\left(A_{k}\right) \wedge A_{n}$, where $A_{k}$ is the most recent compatible revision for $A_{n}$. In other words, $\left(\left(K_{A_{1}}^{*}\right)_{A_{2}}^{*} \cdots\right)_{A_{n}}^{*}=K_{s\left(A_{k}\right) \wedge A_{n}}^{*}$. 
Proof Let $A_{k}$ be the most recent compatible revision for $A_{n}$. Since $A_{n}$ is compatible with the subsequence $A_{1}, \ldots A_{k}$, we have that $\min \left(\left(\left(M_{A_{1}}^{*}\right)_{A_{2}}^{*} \ldots\right)_{A_{k}}^{*}, A_{n}\right)$ lies within the minimal cluster of $\left(\left(M_{A_{1}}^{*}\right)_{A_{2}}^{*} \cdots\right)_{A_{k}}^{*}$. However, each subsequent revision $A_{k+1}, \ldots A_{n-1}$ is incompatible with $A_{n}$ so there can be no $A_{n}$-worlds in the set $\min \left(\left(\left(M_{A_{1}}^{*}\right)_{A_{2}}^{*} \cdots\right)_{A_{i-1}}^{*}, A_{i}\right)$ for $k<i<$ $n$. Since only the relative status of these worlds is changed by these subsequent revisions, an obvious inductive argument (on the number $n \perp k$ of subsequent revisions) shows that $\min \left(\left(\left(M_{A_{1}}^{*}\right)_{A_{2}}^{*} \cdots\right)_{A_{n-1}}^{*}, A_{n}\right)=\min \left(\left(\left(M_{A_{1}}^{*}\right)_{A_{2}}^{*} \cdots\right)_{A_{k}}^{*}, A_{n}\right)$. Since $A_{1}, \ldots A_{k}$ is characterized by $s\left(A_{k}\right)$, the minimal cluster of $\left(\left(M_{A_{1}}^{*}\right)_{A_{2}}^{*} \cdots\right)_{A_{k}}^{*}$ is $\min \left(M, s\left(A_{k}\right)\right)$. Clearly then the minimal cluster of $\left(\left(M_{A_{1}}^{*}\right)_{A_{2}}^{*} \cdots\right)_{A_{n}}^{*}$ is simply $\min \left(M, s\left(A_{k} \wedge A_{n}\right)\right)$. Therefore $A_{1}, \ldots A_{n}$ is characterized by $s\left(A_{k}\right) \wedge A_{n}$.

Theorem 20 For any revision sequence $A_{1}, \ldots A_{n}$, there is some subset of these updates $S \subseteq$ $\left\{A_{1}, \cdots, A_{n}\right\}$ such that $\left(\left(K_{A_{1}}^{*}\right)_{A_{2}}^{*} \cdots\right)_{A_{n}}^{*}=K_{A}^{*}$ and $A=\wedge S$.

Proof This result can be shown using a simple inductive argument on $n$, the number of updates in the sequence. If $n=1$ then the theorem obviously is true, for $A_{1}$ characterizes itself. Now suppose each subsequence $A_{1}, \ldots A_{i}, i<n$, is characterized by some sentence $s\left(A_{i}\right)=\wedge S$, where $S \subseteq\left\{A_{1}, \cdots, A_{i}\right\}$. By Lemma 19 , the sequence $A_{1}, \ldots A_{n}$ is characterized by the sentence $s\left(A_{k}\right) \wedge A_{n}$ for some $k<n$, where $A_{k}$ is the most recent compatible revision for $A_{n}$ (or it is characterized by $A_{n}$ if no such $k$ exists). By the inductive hypothesis, $s\left(A_{n}\right)=\wedge S$ where $S \subseteq\left\{A_{1}, \cdots, A_{k}\right\} \cup\left\{A_{n}\right\} \subseteq\left\{A_{1}, \cdots, A_{n}\right\}$. (If $k=0$ we simple observe that $S=\left\{A_{n}\right\} \subseteq\left\{A_{1}, \cdots, A_{n}\right\}$.)

Theorem 22 Let $A_{1}, \ldots A_{n}$ be a revision sequence with $c$ incompatible updates represented by $\sigma$. For each $1 \leq k \leq n$, if $A_{k}$ is a compatible revision, then $\left(\left(K_{A_{1}}^{*}\right)_{A_{2}}^{*} \cdots\right)_{A_{k}}^{*}=K_{s\left(A_{k}\right)}^{*}$, where: $\left.a\right)$ $\left.s\left(A_{k}\right)=s\left(A_{\sigma(i)}\right) \wedge A_{\sigma(i)+1} \wedge \cdots A_{k} ; b\right) \sigma(i)<k<\sigma(i+1)$, or $\sigma(i)<k$ if $i=c$; and $\left.c\right) s\left(A_{\sigma(i)}\right)$ characterizes subsequence $A_{1}, \ldots A_{\sigma(i)}$.

Proof Let $A_{k}$ be a compatible revision in the sequence $A_{1}, \ldots A_{n}$. If no incompatible revision follows $A_{k}$, then $k>\sigma(i)$, where $i=c$. Otherwise $\sigma(i)<k<\sigma(i+1)$ for some $i<c$. In either case $A_{\sigma(i)}$ is the maximal incompatible revision in the subsequence $A_{1}, \ldots A_{k}$. Let $s\left(A_{\sigma(i)}\right)$ characterize $A_{1}, \ldots A_{\sigma(i)}$. Since each revision $A_{\sigma(i)+1}, \ldots A_{k}$ is compatible, $A_{k-1}$ is the most recent compatible revision for $A_{k}$, and by $k \perp \sigma(i)$ applications of Lemma 19, we have that $A_{1}, \ldots A_{k}$ is characterized by $s\left(A_{k}\right)=s\left(A_{\sigma(i)}\right) \wedge A_{\sigma(i)+1} \wedge \cdots A_{k}$. 
Theorem 24 Let $A_{1}, \ldots A_{n}$ be a revision sequence with c incompatible updates represented by $\sigma$.

For each $1 \leq k \leq n$, if $A_{k}$ is an incompatible revision, then $\left(\left(K_{A_{1}}^{*}\right)_{A_{2}}^{*} \cdots\right)_{A_{k}}^{*}=K_{s\left(A_{k}\right)}^{*}$, where: a) $s\left(A_{k}\right)=s\left(A_{\sigma(i)}\right) \wedge A_{\sigma(i)+1} \wedge \cdots A_{j} \wedge A_{k}$; b) $A_{\sigma(i)}$ is the maximal incompatible revision for $A_{k}$; c) $A_{j}$ is the most recent compatible revision for $A_{k}$; and $\left.d\right) s\left(A_{\sigma(i)}\right)$ characterizes subsequence $A_{1}, \ldots A_{\sigma(i)}$.

Proof Let $A_{j}$ be the most recent compatible revision for $A_{k}$. By Theorem 22 and Proposition 23, $A_{1}, \ldots A_{j}$ is characterized by $s\left(A_{j}\right)=s\left(A_{\sigma(i)}\right) \wedge A_{\sigma(i)+1} \wedge \cdots A_{j}$. By Lemma $19, A_{1}, \ldots A_{k}$ is characterized by $s\left(A_{j}\right) \wedge A_{k}$.

Theorem 30 Let $S_{1}, \cdots S_{n} \in \mathbf{L}_{C P L}$ be such that $\vdash S_{i} \supset \neg S_{j}$ if $i \neq j$, and $\vdash S_{1} \vee \cdots S_{n}$. For all $A, B \in \mathbf{L}_{C P L}$ either

$$
\begin{aligned}
& \operatorname{FSM}\left(S_{1}, \cdots S_{n}\right) \vdash_{C O *} A>B ; \quad \text { or } \\
& \operatorname{FSM}\left(S_{1}, \cdots S_{n}\right) \vdash_{C O *} \neg(A>B)
\end{aligned}
$$

Proof This follows immediately since the theory $\operatorname{FSM}\left(S_{1}, \cdots S_{n}\right)$ is "categorical" in the sense that their is only one $\mathrm{CO}^{*}$-structure satisfying it (modulo "duplicate worlds", which can have no influence on the truth of any sentence in the model).

Theorem 31 Let revision model $M$ be characterized by $F S M\left(S_{1}, \cdots S_{n}\right)$ and let $S_{k}$ be the minimal sentence in this set consistent with $A$; that is, $S_{k} \forall \neg-A$ and $S_{i} \vdash \neg A$ if $i<k$. Then $M_{A}^{*}$ is characterized by

$$
\begin{array}{ll}
F S M\left(S_{k} \wedge A, S_{1}, \cdots S_{k-1}, S_{k} \wedge \neg A, S_{k+1}, \cdots S_{n}\right) & \text { if } S_{k} \not A \\
F S M\left(S_{k}, S_{1}, \cdots S_{k-1}, S_{k+1}, \cdots S_{n}\right) & \text { if } S_{k} \vdash A
\end{array}
$$

Proof Clearly the minimal cluster in $M$ containing $A$-worlds is that cluster specified by the sentence $S_{k}$. Thus the set $\min (M, A)$ consists exactly of those worlds in $M$ satisfying $S_{k} \wedge A$. In $M_{A}^{*}$, this set forms the minimal cluster and all other clusters remain in the same relative order. However, the cluster that was specified by $S_{k}$ is now reduced to those worlds satisfying $S_{k} \wedge \neg A$. If $S_{k} \vdash A$, this is still true; but the sentence $S_{k} \wedge A$ is equivalent to $S_{k}$, and the cluster in $M_{A}^{*}$ satisfying $S_{k} \wedge \neg A$ is empty. 